\title{
Large-Eddy Simulation of Stably Stratified Atmospheric Boundary Layer Turbulence: A Scale-Dependent Dynamic Modeling Approach
}

\author{
Sukanta Basu and Fernando Porté-Agel \\ St. Anthony Falls Laboratory, University of Minnesota, Minneapolis, MN 55414
}

\begin{abstract}
A new tuning-free subgrid-scale model, termed 'locally-averaged scale-dependent dynamic' (LASDD) model, is developed and implemented in large-eddy simulations (LESs) of stable boundary layers. The new model dynamically computes the Smagorinsky coefficient and the subgrid-scale Prandtl number based on the local dynamics of the resolved velocity and temperature fields. Overall, the agreement between the statistics of the LES-generated turbulence and some well-established empirical formulations and theoretical predictions (e.g., Nieuwstadt's local scaling hypothesis) is remarkable. The results show clear improvements over most of the traditional subgrid-scale models in the surface layer. Moreover, in contrast to previous large-eddy simulations of stable boundary layers that have strong dependence on grid resolution, the simulated statistics obtained with the LASDD model show relatively little resolution dependence for the range of grid sizes considered here. In essence, we show that the new LASDD model is a robust subgrid-scale parameterization for reliable, tuning-free simulations of stable boundary layers, even with relatively coarse resolutions.
\end{abstract}

\section{Introduction}

Atmospheric boundary layers (ABLs) are usually classified into three types: neutral, convective and stable, based on atmospheric stability (buoyancy effects) and the dominant mechanism of turbulence generation (Stull 1988; Arya 2001). The boundary layer becomes stably stratified whenever the underlying surface is colder than the air. Under this atmospheric condition, turbulence is generated by shear and destroyed by negative buoyancy and viscosity. Because of this competition between shear and buoyancy effects, the strength of turbulence in the stable boundary layer (SBL) is much weaker in comparison to the neutral and convective boundary layers. As a result, the stable boundary layer is also much shallower and characterized by smaller eddy motions. Stable boundary layer turbulence has not received much attention despite its scientifically intriguing nature and practical significance (e.g., numerical weather prediction NWP, and pollutant transport). This might be attributed to the lack of adequate field or laboratory measurements, to the inevitable difficulties in numerical simulations (arising from small scales of motion due to stratification) and to the intrinsic complexity in its dynamics (e.g., occurrences of intermittency, Kelvin-Helmholtz instability, gravity waves, low-level jets, meandering motions etc.) (Hunt et al. 1996;

Corresponding author address:

Sukanta Basu, St. Anthony Falls Laboratory, University of Minnesota, Minneapolis, MN 55414 (basus@msi.umn.edu)
Mahrt 1998; Derbyshire 1999). Not surprisingly, today, there is a general consensus among researchers that our understanding of the stable boundary layer (especially the very stable regime) is quite poor (Mahrt 1998; Derbyshire 1999; Holtslag 2003) and 'even small future advances justify more work' (Mahrt 1998).

In order to improve our understanding of SBL turbulence and to explore some of its inherent characteristics, in this study we make use of a contemporary numerical modeling approach, known as large-eddy simulation. Following the pioneering works of Deardorff (Deardorff 1970, 1972, 1974, 1980) over the years LES has become an indispensable tool to study the ABL (e.g., Moeng 1984; Nieuwstadt et al. 1991; Andrén et al. 1994; Mason 1994; Sullivan et al. 1994; Kosović 1997; Albertson and Parlange 1999; Porté-Agel et al. 2000; Beare et al. 2004). However, until now LES models have not been sufficiently faithful in reproducing the characteristics of moderately and strongly stable atmospheric boundary layers (Saiki et al. 2000; Holtslag 2003). The main weakness of LES is associated with our limited ability to accurately account for the dynamics that are not explicitly resolved in the simulations. Under stable conditions - due to flow stratification - the characteristic size of the eddies becomes increasingly smaller with increasing atmospheric stability, which eventually imposes an additional burden on the LES subgrid-scale (SGS) models. The recent GABLS (Global Energy and Water Cycle Experiment Atmospheric Boundary Layer Study) LES intercomparison 
study (Beare et al. 2004) highlights that LESs of moderately stable BLs are quite sensitive to SGS models at a relatively fine resolution of $6.25 \mathrm{~m}$. At a coarser resolution $(12.5 \mathrm{~m})$, a couple of commonly used SGS models even laminarized spuriously. Occasionally, laminarization was manifested by a near-linear (without any curvature) temperature profile; at times the SGS contributions to the total momentum or heat fluxes were larger than fifty percent in the interior of the boundary layer. This breakdown of traditional SGS models undoubtedly calls for improved SGS parameterizations in order to make LES a more reliable tool to study stable boundary layers. The present study is devoted towards this goal.

The organization of this paper is as follows. In Section 2, we briefly describe the basic philosophy of large-eddy simulation. The newly developed locally-averaged scaledependent dynamic (LASDD) SGS modeling approach is presented in Section 3. Simulations of stably stratified atmospheric boundary layers are presented in Section 4. Lastly, in Section 5, we summarize our research and elaborate on the prospects for future research in this subject area.

\section{Subgrid-Scale Modeling and SGS Parameter Estimation}

In rotation-influenced ABLs, the equations governing the conservation of momentum (using the Boussinesq approximation) and temperature are:

$$
\begin{gathered}
\frac{\partial \tilde{u}_{i}}{\partial t}+\frac{\partial\left(\tilde{u}_{i} \tilde{u}_{j}\right)}{\partial x_{j}}=-\frac{\partial \tilde{p}}{\partial x_{i}}-\frac{\partial \tau_{i j}}{\partial x_{i}}+\delta_{i 3} g \frac{(\tilde{\theta}-\langle\tilde{\theta}\rangle)}{\theta_{0}}+f_{c} \epsilon_{i j 3} \tilde{u}_{j}+F_{i} \\
\frac{\partial \tilde{\theta}}{\partial t}+\frac{\partial\left(\tilde{u}_{j} \tilde{\theta}\right)}{\partial x_{j}}=-\frac{\partial q_{j}}{\partial x_{j}}
\end{gathered}
$$

where $t$ is time, $x_{j}$ is the spatial coordinate in the $j$-direction, $u_{j}$ is the velocity component in that direction, $\theta$ is potential temperature, $\theta_{0}$ is reference surface potential temperature, $p$ is dynamic pressure, $\delta_{i 3}$ is the Kronecker delta, $\epsilon_{i j 3}$ is the alternating unit tensor, $g$ is the gravitational acceleration, $f_{c}$ is the Coriolis parameter and $F_{i}$ is a forcing term (e.g., Geostrophic wind or imposed mean pressure gradient). Molecular dissipation and diffusion have been neglected since the Reynolds number of the ABL is very high and no near-ground viscous processes are resolved. The \langle\rangle is used to define a horizontal plane average. The $\widetilde{(\cdots)}$ denotes a spatial filtering operation, using a filter of characteristic width $\Delta_{f}$. These filtered equations are now amenable for numerical solution (LES) on a grid of mesh size $\Delta_{g}$, considerably larger than the smallest scale of turbulent motion (the so-called Kolmogorov scale). It is common practice to use the ratio of filter-width to grid-spacing, $\Delta_{f} / \Delta_{g}=1$ or 2 [see the Chapter 9 of Geurts (2003) for detailed discussion on this ratio and its impact on error dynamics]. In this study, we use a ratio of 2 .
The effects of the unresolved scales (smaller than $\Delta_{f}$ ) on the evolution of $\tilde{u}_{i}$ and $\tilde{\theta}$ appear in the SGS stress $\tau_{i j}$ (see Equation 1) and the SGS flux $q_{i}$ (see Equation 2), respectively. They are defined as

$$
\tau_{i j}=\widetilde{u_{i} u_{j}}-\tilde{u}_{i} \tilde{u}_{j}
$$

and

$$
q_{i}=\widetilde{u_{i} \theta}-\tilde{u}_{i} \tilde{\theta}
$$

Note that the SGS stress and flux quantities are unknown and must be parameterized (using a SGS model) as a function of the resolved velocity and temperature fields.

Eddy-viscosity models, the most popular SGS models, use the 'gradient hypothesis' and formulate the $i j$-component of the SGS stress tensor (the deviatoric part) as follows (Smagorinsky 1963; Geurts 2003):

$$
\tau_{i j}-\frac{1}{3} \tau_{k k} \delta_{i j}=-2 \nu_{t} \tilde{S}_{i j},
$$

where

$$
\tilde{S}_{i j}=\frac{1}{2}\left(\frac{\partial \tilde{u}_{i}}{\partial x_{j}}+\frac{\partial \tilde{u}_{j}}{\partial x_{i}}\right)
$$

is the resolved strain rate tensor and $\nu_{t}$ denotes the eddyviscosity. It is well-known that the eddy-viscosity SGS models give a poor prediction of the SGS stresses on a local level (see Sarghini et al. 1999; Meneveau and Katz 2000; Geurts 2003.) Their underlying assumption of strain rates being aligned with the SGS stress tensor is unrealistic (see Higgins et al. 2003 and the references therein). Furthermore, these SGS models are purely dissipative, i.e., they do not allow local reverse energy transfer (known as 'backscatter') (Sarghini et al. 1999). Despite all these deficiencies, without any doubt, the eddy-viscosity models are the most commonly used SGS models in the atmospheric boundary layer community.

From dimensional analysis, the eddy-viscosity $\left(\nu_{t}\right)$ can be interpreted as the product of a characteristic velocity scale and a characteristic length scale (Geurts 2003). Different eddy-viscosity formulations basically use different velocity and length scales. The most popular eddy-viscosity formulation is the Smagorinsky model (Smagorinsky 1963):

$$
\nu_{t}=\left(C_{S} \Delta_{f}\right)^{2}|\tilde{S}|,
$$

where $C_{S}$ is the so-called Smagorinsky coefficient, which is adjusted empirically or dynamically to account for shear, stratification and grid-resolution, and

$$
|\tilde{S}|=\left(2 \tilde{S}_{i j} \tilde{S}_{i j}\right)^{1 / 2}
$$

is the magnitude of the resolved strain rate tensor. In contrast to the Smagorinsky-type eddy-viscosity model, the turbulence kinetic energy (TKE) based eddy-viscosity model 
utilizes (Moeng 1984; Sullivan et al. 1994; Sullivan et al. 2003):

$$
\nu_{t}=C_{K} l E_{S G S}^{1 / 2},
$$

where $C_{K}$ is a modeling coefficient, $l$ is a length scale and $E_{S G S}$ is the SGS turbulence kinetic energy. This modeling approach involves solving an extra prognostic equation for the SGS TKE. Based on the Kolmogorov's scaling laws, Wong and Lilly (1994) proposed yet another eddy-viscosity model:

$$
\nu_{t}=C^{2 / 3} \Delta_{f}^{4 / 3} \epsilon^{1 / 3}=C_{\epsilon} \Delta_{f}^{4 / 3},
$$

where $\epsilon$ is the dissipation rate of energy and $C_{\epsilon}$ is a model coefficient to be specified (or determined dynamically). There are numerous other formulations for eddy-viscosity existing in the literature (e.g., the Structure Function model of Métais and Lesieur 1992). An extensive review of these formulations is given by Sagaut (2001).

Similar to the SGS stresses, the SGS heat fluxes are modeled with the eddy-diffusivity models as:

$$
q_{i}=-\nu_{h t} \frac{\partial \tilde{\theta}}{\partial x_{i}}=-\frac{\nu_{t}}{P r_{S G S}} \frac{\partial \tilde{\theta}}{\partial x_{i}}
$$

where $P r_{S G S}$ is the SGS Prandtl number.

The values of the Smagorinsky-type SGS model parameters $C_{S}$ and $P r_{S G S}$ are well established for homogeneous, isotropic turbulence (Lilly 1967). However, the value of $C_{S}$ is expected to decrease with increasing mean shear and stratification. This has been confirmed by various recent field studies (Porté-Agel et al. 2001; Kleissl et al. 2003; Sullivan et al. 2003; Kleissl et al. 2004). In order to account for this, application of the traditional eddy-viscosity model in LES of ABL flows (with strong shear near the ground and temperature-driven stratification) has traditionally involved the use of various types of wall-damping functions and stability corrections, which are either based on the phenomenological theory of turbulence or empirically derived from observational data (Mason 1994). For example, recently, based on the HATS (Horizontal Array Turbulence Study) field campaign data Kleissl et al. (2003, 2004) proposed the following empirical form for $C_{S}$ :

$$
\left(C_{S}\right)_{\Delta_{f}}=c_{0}\left[1+R\left(\frac{\Delta_{f}}{L}\right)\right]^{-1}\left[1+\left(\frac{c_{0}}{\kappa} \frac{\Delta_{f}}{z}\right)^{n}\right]^{1 / n},
$$

where $L$ is the Obukhov length, $\kappa$ is the von Karman constant, $R$ is the ramp function, $n=3$ and $c_{0} \approx 0.135$. Another example would be Smagorinsky-type SGS models that impose both the wall-damping and stability corrections based on the Kansas field experiment data (see Brown et al. 1994; Mason 1994; Mason and Brown 1999; Beare and MacVean 2004). In the case of TKE-based eddy-viscosity models, the length scale $l$ is usually set equal to the filter width $\Delta_{f}$ for unstable and neutral stratifications and equal to
$C_{l} \sqrt{E_{S G S}} / N$ for stable stratification (Sullivan et al. 1994; Saiki et al. 2000; Sullivan et al. 2003), following the suggestion of Deardorff (1980). Here, $C_{l}$ is a coefficient to be prescribed and $N$ is the Brunt-Väisälä frequency. When using this approach, the SGS model coefficients are often 'tuned' for different ABL flow conditions (Sullivan et al. 1994; Saiki et al. 2000; Sullivan et al. 2003). There also have been a few elegant attempts to derive shear and stability dependent length-scales directly from the phenomenological theory of turbulence (Hunt et al. 1988; Schumann 1991; Canuto and Cheng 1997). 'The adequacy of all these parameterizations for SGS fluxes remains relatively untested however' (Sullivan et al. 2003).

In the case of eddy-diffusivity SGS models, one needs to prescribe the stability dependence of the SGS Prandtl number $\left(P r_{S G S}\right)$. In general, $\operatorname{Pr}_{S G S}$ is found to increase under stable stratification, which is reflected in different SGS modeling approaches. For example, in a widely used Smagorinsky-type SGS model, the Prandtl number is increased from 0.44 in the free convection limit to 0.7 in neutral condition to 1.0 in the very stable regime (Mason and Brown 1999). In contrast, the TKE-based SGS model uses (Moeng 1984; Sullivan et al. 1994; Saiki et al. 2000; Sullivan et al. 2003): $P r_{S G S}=\Delta_{f} /\left(\Delta_{f}+2 l\right)$, where $l$ is defined as before. This implies that the $P r_{S G S}$ is 0.33 under convective and neutral conditions and varies from 0.33 (weakly stable) to 1.0 (very stable) in the stably stratified regime.

In summary, most of the conventional eddy-viscosity and eddy-diffusivity SGS modeling approaches involve parameter tuning or a priori prescription in one way or another. An alternative approach is to use the 'dynamic' SGS modeling approach of Germano (Germano et al. 1991; Germano 1992; Lilly 1992). In this approach, one computes the value of the unknown SGS coefficient (e.g., the coefficient $C_{S}$ in the Smagorinsky-type eddy-viscosity models) dynamically at every time and position in the flow. By looking at the dynamics of the flow at two different resolved scales and assuming scale similarity as well as scale invariance of the model coefficient, one can optimize its value. Thus, the dynamic model avoids the need for a priori specification and tuning of the coefficient because it is evaluated directly from the resolved scales in an LES. In Moin et al. (1991), a similar dynamic procedure was applied to estimate the SGS scalar flux in compressible flows. In essence this procedure not only eliminates the need for any ad-hoc assumption about the SGS Prandtl number $\left(\operatorname{Pr}_{S G S}\right)$ but also completely decouples the SGS flux estimation from SGS stress computation, which is highly desirable. 


\section{Locally-Averaged Scale-Dependent Dynamic Modeling Approach}

In the previous section, we mentioned that the SGS stress tensor $\left(\tau_{i j}\right)$ at the filter scale $\left(\Delta_{f}\right)$ is defined as: $\tau_{i j}=$ $\widetilde{u_{i} u_{j}}-\widetilde{u_{i}} \widetilde{u_{j}}$. In a seminal work, Germano (Germano et al. 1991; Germano 1992) proposed to invoke an additional explicit test filter of width $\alpha \Delta_{f}$ in order to dynamically compute the SGS coefficients. Consecutive filtering at scales $\Delta_{f}$ and at $\alpha \Delta_{f}$ leads to a SGS turbulent stress tensor $\left(T_{i j}\right)$ at the test filter scale $\alpha \Delta_{f}$ :

$$
T_{i j}=\overline{\overline{u_{i} u_{j}}}-\overline{\widetilde{u}_{i}} \overline{\widetilde{u}_{j}}
$$

where an overline $\overline{(\cdots)}$ denotes filtering at a scale of $\alpha \Delta_{f}$. From the definitions of $\tau_{i j}$ and $T_{i j}$ an algebraic relation can be formed, known in the literature as the Germano identity:

$$
L_{i j}=\overline{\widetilde{u}_{i} \widetilde{u}_{j}}-\overline{\widetilde{u}_{i}} \overline{\widetilde{u}_{j}}=T_{i j}-\overline{\tau_{i j}}
$$

This identity is then effectively used to dynamically obtain unknown SGS model coefficients. In the case of the Smagorinsky model, this identity yields ${ }^{1}$ :

$$
L_{i j}-\frac{1}{3} L_{k k} \delta_{i j}=\left(C_{S}^{2}\right)_{\Delta_{f}} M_{i j}
$$

where $M_{i j}=2 \Delta_{f}^{2}\left(\overline{|\widetilde{S}| \widetilde{S_{i j}}}-\alpha^{2} \frac{\left(C_{S}^{2}\right)_{\alpha \Delta_{f}}}{\left(C_{S}^{2}\right)_{\Delta_{f}}}|\widetilde{\widetilde{S}}| \overline{\widetilde{S_{i j}}}\right)$. If one assumes scale invariance, i.e., $\left(C_{S}^{2}\right)_{\alpha \Delta_{f}}=\left(C_{S}^{2}\right)_{\Delta_{f}}$ (Germano et al. 1991), then the unknown coefficient $\left(C_{S}^{2}\right)_{\Delta_{f}}$ can be easily determined following the error minimization approach of Lilly (1992):

$$
\left(C_{S}^{2}\right)_{\Delta_{f}}=\frac{\left\langle L_{i j} M_{i j}\right\rangle}{\left\langle M_{i j} M_{i j}\right\rangle}
$$

Here the angular brackets $\langle\cdots\rangle$ denote spatial averaging (Germano et al. 1991; Lilly 1992; Zang et al. 1993; Ghosal et al. 1995; Porté-Agel et al. 2000) or averaging over fluid pathlines (Meneveau et al. 1996).

In a recent study, Porté-Agel et al. (2000) found that in a simulation of neutral boundary layer the scale-invariant dynamic model is not dissipative enough in the near-ground region. It underestimates shear in that region and also yields excessively flat spectra (Porté-Agel et al. 2000). Moreover, the dynamically computed coefficient $C_{S}^{2}$ show clear scaledependence near the surface, i.e., $\left(C_{S}^{2}\right)_{\Delta_{f}} \neq\left(C_{S}^{2}\right)_{\alpha \Delta_{f}}$. Similar inferences are also obtained in the case of passive scalars (Porté-Agel 2004). Field observations by Kleissl et al. (2004) also support these results.

\footnotetext{
${ }^{1}$ Please note that here the variation of $C_{S}^{2}$ over the test filter scale has been implicitly neglected (Germano et al. 1991; Lilly 1992; Vreman et al. 1994).
}

This prompted Porté-Agel et al. (2000) to propose the scale-dependent dynamic SGS model. In this case, the scaledependence parameter $\beta=\frac{\left(C_{S}^{2}\right)_{\alpha \Delta_{f}}}{\left(C_{S}^{2}\right)_{\Delta_{f}}}$ is not assumed to be equal to one, rather it is determined dynamically. In order to implement this procedure, one needs to employ a second test filtering operation at a scale of $\alpha^{2} \Delta_{f}$ [denoted by $\left.\widehat{(\cdots)}\right]$. Invoking the Germano identity for the second time leads to:

$$
Q_{i j}-\frac{1}{3} Q_{k k} \delta_{i j}=\left(C_{S}^{2}\right)_{\Delta_{f}} N_{i j}
$$

where

$$
Q_{i j}=\widehat{\widetilde{u_{i}}}{\widetilde{u_{j}}}-\widehat{\tilde{u}_{i}}
$$

and

$$
N_{i j}=2 \Delta_{f}^{2}\left(|\widehat{\widetilde{S}}| \widetilde{S_{i j}}-\alpha^{4} \frac{\left(C_{S}^{2}\right)_{\alpha^{2} \Delta_{f}}}{\left(C_{S}^{2}\right)_{\Delta_{f}}}|\widehat{\widetilde{S}}| \widehat{\widetilde{S_{i j}}}\right)
$$

This results in:

$$
\left(C_{S}^{2}\right)_{\Delta_{f}}=\frac{\left\langle Q_{i j} N_{i j}\right\rangle}{\left\langle N_{i j} N_{i j}\right\rangle}
$$

In the scale-dependent dynamic modeling approach, the following assumption is made:

$$
\beta=\frac{\left(C_{S}^{2}\right)_{\alpha \Delta_{f}}}{\left(C_{S}^{2}\right)_{\Delta_{f}}}=\frac{\left(C_{S}^{2}\right)_{\alpha^{2} \Delta_{f}}}{\left(C_{S}^{2}\right)_{\alpha \Delta_{f}}}
$$

which is a much weaker assumption than the scale-invariance modeling assumption of $\beta=1$. Now, from Equations 14 and 16 , one solves for the unknown parameter $\beta$, which in turn is used to compute $\left(C_{S}^{2}\right)_{\Delta_{f}}$ utilizing Equation 14. Further technical details on this model could be found in PortéAgel et al. (2000). In Porté-Agel (2004), similar formulations were derived for scalars. In this case, the lumped SGS coefficient $C_{S}^{2} \operatorname{Pr}_{S G S}^{-1}$ was determined dynamically and the scale-dependent parameter was termed as $\beta_{\theta}$. In the simulations of neutral boundary layers, the scale-dependent SGS model was found to exhibit 'right' dissipation behavior and more accurate spectra in the case of momentum (Porté-Agel et al. 2000), as well as for passive scalars (Porté-Agel 2004).

In the present study we found that the original formulation of Porté-Agel et al. (2000) which involves (horizontal) planar averaging in Equation 14, suffers from an insufficient SGS dissipation problem in the outer layers in simulations of stable boundary layers. This could be attributed to decoupling between horizontal planes under stratification. Intermittent, patchy turbulence in the strongly stable outer layers might be another cause. This issue was resolved by using a local formulation of the scale-dependent modeling approach, named as the locally-averaged scale-dependent dynamic (LASDD) model. The model coefficients $\left(C_{S}^{2}\right.$ and 
$C_{S}^{2} \operatorname{Pr}_{S G S}^{-1}$ ) are obtained dynamically by averaging locally on the horizontal plane with a stencil of three by three grid points. Zang et al. (1993) followed a similar approach in the scale-invariant dynamic (i.e., $\beta=1$ ) modeling of turbulent recirculating flows. To avoid numerical instabilities the coefficients $C_{S}^{2}$ and $C_{S}^{2} \operatorname{Pr}_{S G S}^{-1}$ are set to zero whenever the dynamic procedure yields negative values. This commonly used procedure is known as 'clipping' (Geurts 2003). The scale-dependence parameters ( $\beta$ and $\beta_{\theta}$ ) are determined dynamically over horizontal planes to avoid the computational burden of computing them at every grid point in the flow. Solving for $\beta$ or $\beta_{\theta}$ involves a fifth-order polynomial. Instead of the Newton-Raphson method used by Porte-Agel et al. (2000) and Porté-Agel (2004), we use a more robust eigenvalue based method (Press et al. 1992) to obtain the roots of this polynomial. In the infrequent events that an appropriate real root in the range of 0 to 1.2 is not found, we chose to invoke the scale-invariance assumption of $\beta$ (or, $\beta_{\theta}$ ) $=1$.

Please note that, mathematically more rigorous (and computationally more expensive) local models are also proposed in the literature (Ghosal et al. 1995; Piomelli and Liu 1995; Meneveau et al. 1996). Their capabilities in the stably stratified atmospheric boundary layer simulations have yet to be tested.

\section{LES of Stably Stratified Boundary Layers}

The first LES of a stable boundary layer was performed by Mason and Derbyshire (1990). They used the traditional Smagorinsky-type SGS model with a constant SGS Prandtl number $\left(\operatorname{Pr}_{S G S}=0.5\right)$. Their results broadly supported the local scaling hypothesis of Nieuwstadt (Nieuwstadt 1984a; Nieuwstadt 1984b; Nieuwstadt 1985; Derbyshire 1990). However, one of their simulations showed the run-away cooling problem. The simulated surface temperature fell more than $30 \mathrm{~K}$ over 90 minutes. Brown et al. (1994) repeated these simulations with their stochastic backscatter SGS model (with stability corrections). Their results definitely showed some improvements (especially in the surface layer properties) when compared with the Mason and Derbyshire's simulations.

Andrén (1995) simulated weakly stable boundary layers with the TKE-based SGS model of Moeng (1984) and also with the two-part eddy-viscosity model developed by Sullivan et al. (1994). The two-part eddy-viscosity model, which is a modified version of the TKE-based SGS model, was in better agreement with the surface-layer similarity theory.

Recently, Saiki et al. (2000) attempted to simulate a moderately stable boundary layer with Sullivan et al.'s two-part eddy-viscosity model (Sullivan et al. 1994). Although, this particular SGS scheme has been previously used by Andrén for weakly stable BLs (Andrén 1995), in the case of mod- erately stable boundary layers it led to development of unphysical profiles of various turbulent quantities Saiki et al. 2000). The failure was due to the collapse of SGS vertical heat flux near the surface. This prompted them to propose a two-part SGS model to represent the SGS heat flux similar to the SGS momentum flux model of Sullivan et al. (1994). However, even after the modifications, the simulations were found to be too sensitive to rapid cooling (Saiki et al. 2000).

Kosović and Curry (2000) simulated a clear-air, moderately stable boundary layer as it approaches quasi-steady state using the nonlinear SGS model of Kosović (1997). Initial conditions consistent with the Beaufort Sea Arctic Stratus Experiment (BASE) observations were used. The first intercomparison study of LES-SGS models for stable boundary layer (Holtslag 2003; Beare et al. 2004), as part of the GABLS initiative, also used this case (slightly modified). Eleven different models with very different SGS modeling options and different grid-resolutions (from $1 \mathrm{~m}$ to $12.5 \mathrm{~m}$ ) were run (Beare et al. 2004). In this paper, we also simulate this particular case with our newly proposed locally-averaged scale-dependent dynamic (LASDD) SGS model. We compare our results with theoretical predictions by Nieuwstadt (Nieuwstadt 1984a, b, 1985), field observations, as well as with well-established empirical formulations.

\section{a. Description of the Simulations}

The GABLS LES intercomparison case study is described in detail in Beare et al. (2004). The boundary layer is driven by an imposed, uniform geostrophic wind $(G=8$ $\mathrm{m} \mathrm{s}^{-1}$ ), with a surface cooling rate of $0.25 \mathrm{~K}$ per hour and attains a quasi-steady state in $\sim 8-9$ hours with a boundary layer depth of $\sim 200 \mathrm{~m}$. The initial mean potential temperature is $265 \mathrm{~K}$ upto $100 \mathrm{~m}$ with an overlying inversion of strength $0.01 \mathrm{~K} \mathrm{~m}^{-1}$. The Coriolis parameter is set to $f_{c}=1.39 \times 10^{-4} \mathrm{~s}^{-1}$, corresponding to latitude $73^{\circ} \mathrm{N}$. Our domain size is: $\left(L_{x}=L_{y}=L_{z}=400 \mathrm{~m}\right)$. This domain is divided into: (1) $N_{x} \times N_{y} \times N_{z}=32 \times 32 \times 32$ nodes (i.e., $\Delta_{x}=\Delta_{y}=\Delta_{z}=12.5 \mathrm{~m}$ ); (2) $N_{x} \times N_{y} \times N_{z}=$ $64 \times 64 \times 64$ nodes (i.e., $\Delta_{x}=\Delta_{y}=\Delta_{z}=6.25$ m); and (3) $N_{x} \times N_{y} \times N_{z}=80 \times 80 \times 80$ nodes (i.e., $\Delta_{x}=\Delta_{y}=\Delta_{z}=5 \mathrm{~m}$ ). One of the objectives behind these simulations was to investigate the sensitivity of our results to grid resolution.

A Galilean transformation is used to weaken the stability constraints on the time step. The grid moves with $\left(U_{\text {Gal }}, V_{\text {Gal }}\right)=(5.5,0) \mathrm{m} / \mathrm{s}$ in the $32^{3}$ and $64^{3}$ nodes cases. In the case of $80^{3}$ simulation, we have used $\left(U_{\text {Gal }}, V_{\text {Gal }}\right)=$ $(5,0) \mathrm{m} / \mathrm{s}$. The time steps $(\Delta t)$ for our $32^{3}, 64^{3}$, and $80^{3}$ simulations are $0.4,0.2$ and 0.14 seconds, respectively. 


\section{b. Description of the LES Code}

In this work, we have used a modified version of the LES code described in Albertson and Parlange (1999), PortéAgel et al. (2000), and Porté-Agel (2004). The salient features of this code are as follows:

- It solves the filtered Navier-Stokes equations written in rotational form (Orszag and Pao 1974).

- Derivatives in the horizontal directions are computed using the Fourier Collocation method, while vertical derivatives are approximated with second-order central differences (Canuto et al. 1988).

- Dealiasing of the nonlinear terms in Fourier space is done using the $3 / 2$ rule (Canuto et al. 1988).

- Explicit second-order Adams-Bashforth time advancement scheme is used (Canuto et al. 1988).

- Explicit spectral cutoff filtering is used. The ratio between the filter-width $\left(\Delta_{f}\right)$ and grid-spacing $\left(\Delta_{g}\right)$ is set to 2 . Here $\alpha$ is taken to be equal to $\sqrt{2}$.

- Only Coriolis terms involving horizontal wind are considered.

- Forcing is imposed by Geostrophic wind.

- Staggered vertical grid is used.

The lower boundary condition is based on the MoninObukhov similarity theory with a surface roughness length $z_{\circ}$. The instantaneous components of surface shear stresses $\tau_{x z}$ and $\tau_{y z}$ are represented as functions of the resolved velocities $\tilde{u}$ and $\tilde{v}$, at the grid point immediately above the surface (i.e., at a height of $z=\Delta_{z} / 2$ in our case):

$$
\begin{gathered}
\tau_{x z}=-u_{*}^{2}\left[\frac{\tilde{u}(z)}{U(z)}\right] \\
\tau_{y z}=-u_{*}^{2}\left[\frac{\tilde{v}(z)}{U(z)}\right],
\end{gathered}
$$

where $u_{*}$ is the friction velocity, which is computed from the mean horizontal wind speed $U(z)=\left\langle\left(\tilde{u}^{2}+\tilde{v}^{2}\right)^{1 / 2}\right\rangle$ at the first vertical model level $\left(z=\Delta_{z} / 2\right)$ as follows:

$$
u_{*}=\frac{U(z) \kappa}{\log \left(\frac{z}{z_{\circ}}\right)+b_{m} \frac{z}{L}} .
$$

In a similar manner, the surface heat flux is computed as:

$$
\left\langle w \theta_{s}\right\rangle=\frac{u_{*} \kappa\left[\theta_{s}-\Theta(z)\right]}{\log \left(\frac{z}{z_{\circ}}\right)+b_{h} \frac{z}{L}},
$$

where $\theta_{s}$ and $\Theta(z)$ denote the surface temperature and the mean resolved potential temperature at the first model level,
TABLE 1. Basic characteristics of the simulated SBLs during the last hour of simulation.

\begin{tabular}{lcccc}
\hline \hline Grid Points & $H(\mathrm{~m})$ & $L(\mathrm{~m})$ & $u_{*}\left(\mathrm{~ms}^{-1}\right)$ & $\theta_{*}(\mathrm{~K})$ \\
\hline $32 \times 32 \times 32$ & 205 & 113 & 0.283 & 0.047 \\
$64 \times 64 \times 64$ & 185 & 114 & 0.276 & 0.045 \\
$80 \times 80 \times 80$ & 192 & 122 & 0.285 & 0.045 \\
\hline
\end{tabular}

respectively. Following the recommendations of the GABLS intercomparison study, the constants $b_{m}$ and $b_{h}$ were set to 4.8 and 7.8 , respectively.

The upper boundary consists of a zero stress condition, whereas the lateral boundary condition assumes periodicity. A Rayleigh damping layer at $300 \mathrm{~m}$ is used following the GABLS case description.

\section{c. Results}

In this section we report the results of our tuning-free simulations and attempt to evaluate them against the theoretical predictions and well established observations-based formulations. Our results show clear improvements over most of the traditional models in the surface layer. We would like to emphasize that in the surface layer the relative contribution of the SGS to the overall turbulent fluxes is very large. This is also the location where gradients are much stronger. Hence, simulation results become very sensitive to SGS formulations near the ground. The comparisons we perform are extensive and address: (1) temporal evolution of simulated statistics, (2) various first order statistics of turbulent velocity and temperature fields, (3) second order statistics of turbulent velocity and temperature fields, and (4) characteristics of dynamically estimated SGS coefficients. Details of these comparisons are provided below.

The boundary layer height $(H)$, Obukhov length $(L)$ and other characteristics of the simulated SBLs using the locallyaveraged scale-dependent dynamic SGS model (averaged over the final hour of simulation) are given in Table 1. Following Kosović and Curry (2000) and Beare et al. (2004), the boundary layer height $(H)$ is defined as $(1 / 0.95)$ times the height where the mean local stress falls to five percent of its surface value. From this table, it is apparent that the simulated (bulk) boundary layer parameters are quite insensitive to the grid resolution. In LES this behavior is always desirable and its existence is usually attributed to the strength of a SGS model.

\section{1) TEMPoral EVOLUTiON}

In Figures 1 and 2 the time series of surface momentum flux, surface buoyancy flux, Obukhov length $(L)$ and boundary layer height $(H)$ are shown. The surface momentum flux reaches quasi-equilibrium after 4 hours of simulation. On the other hand the Obukhov length and boundary layer 

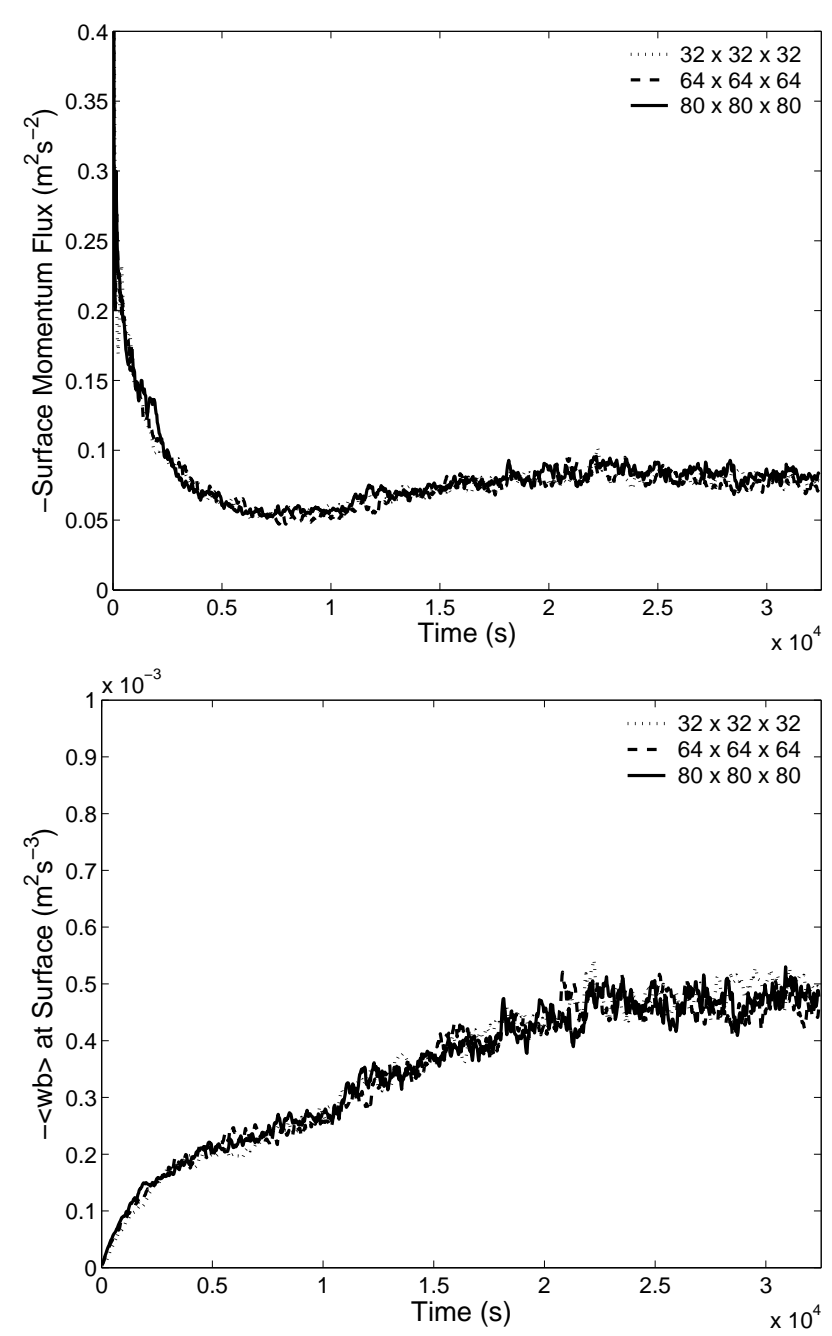

FIG. 1. Time series of surface momentum flux (top) and surface buoyancy flux (bottom).

height equilibrate well before 2 hours of simulation. Since the surface boundary condition is prescribed by a fixed cooling rate rather than a fixed flux, it is anticipated that the surface buoyancy flux will keep on evolving with time.

\section{2) First-ORDER STATISTICS}

The mean profiles of wind speed, wind angle and potential temperature averaged over the final hour (8-9 hours) of simulation, are shown in Figures 3 and 4. The supergeostrophic nocturnal jet near the top of the boundary layer, is in accordance with Nieuwstadt's theoretical model for 'stationary' stable boundary layers [see Equation 17 of Nieuwstadt (1985)]. However, the angle between the surface wind direction and the geostrophic wind simulated by our LES is $\sim 35$ degrees. This is much smaller than Nieuwstadt's prediction of 60 degrees. The second-order closure model of Brost and Wyngaard (1978) also predicts a value of $\sim 58$ de-
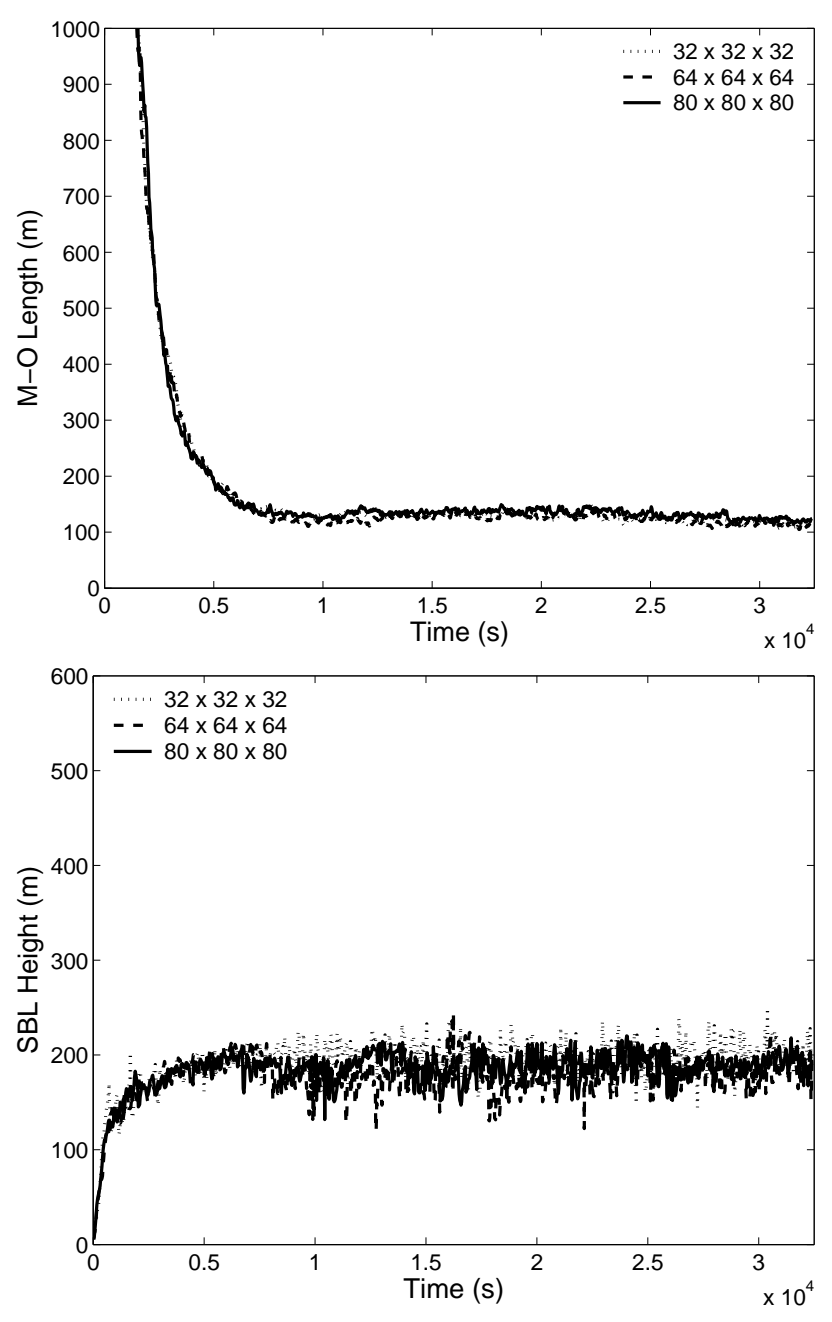

FIG. 2. Time series of Obukhov length (top) and boundary layer height (bottom).

grees. In contrast, the results from the GABLS study (Beare et al. 2004) and Kosović and Curry (2000) are in agreement with our results.

Nieuwstadt also derived the following mean temperature profile [Equation 21 of Nieuwstadt (1985)]:

$$
\frac{\Theta-\theta_{s}}{\theta_{*}}=-\frac{R i_{g}}{\kappa R i_{f}^{2}} \frac{H}{L} \ln \left(1-\frac{z}{H}\right),
$$

where $R i_{f}$ and $R i_{g}$ denote the flux and the gradient Richardson numbers, respectively. $\theta_{*}\left(=-\frac{\langle w \theta\rangle}{u_{*}}\right)$ signifies the surface layer temperature scale. Equation 22 implies that the temperature profile exhibits positive curvature $\left(\sim \partial^{2} \Theta / \partial z^{2}\right)$, which is clearly visible in Figure 4.

We would like to point out that Nieuwstadt's analytical model is based on the hypothesis that the gradient Richardson number $\left(R i_{g}\right)$ and the flux Richardson number $\left(R i_{f}\right)$ are constant with height inside the stable boundary layer. 

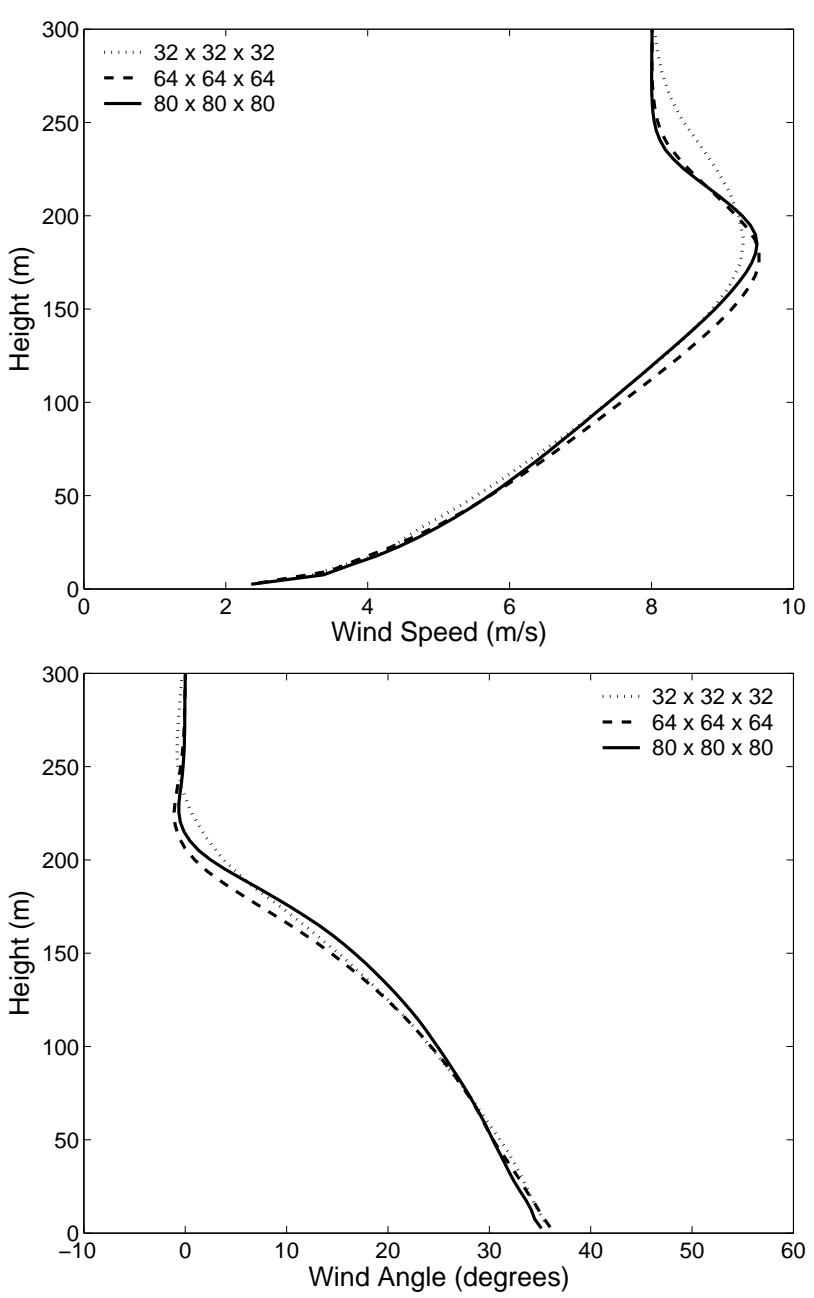

FIG. 3. Mean wind speed (top) and wind angle (bottom) profiles. These profiles are averaged over the last one hour of simulation.

Nieuwstadt was aware of the fact that this hypothesis does not hold for the lower part of the boundary layer (Nieuwstadt 1985). In fact, $R i_{g}$ and $R i_{f}$ should go to zero near the surface (Nieuwstadt 1985), as can be seen from our simulations (Figure 5). The violation of the basic assumption in the proximity of the land surface might explain some of the discrepancies between the LES results and Nieuwstadt's predictions.

The Richardson numbers represent the ratio of the amount of turbulent kinetic energy (TKE) destroyed by buoyancy forces to the amount of TKE generated by wind shear (Stull 1988). The values of $R i_{f}$ are consistently higher than the corresponding $R i_{g}$ values, which is expected (see below). In the interior part of the boundary layer, $R i_{g}$ is more or less constant $(\sim 0.2)$, in accord with Nieuwstadt's assumption. However, $R i_{f}$ increases monotonically and is higher than 0.2 in the upper part of the boundary layer. The magnitudes of both these Richardson numbers increase sharply near the

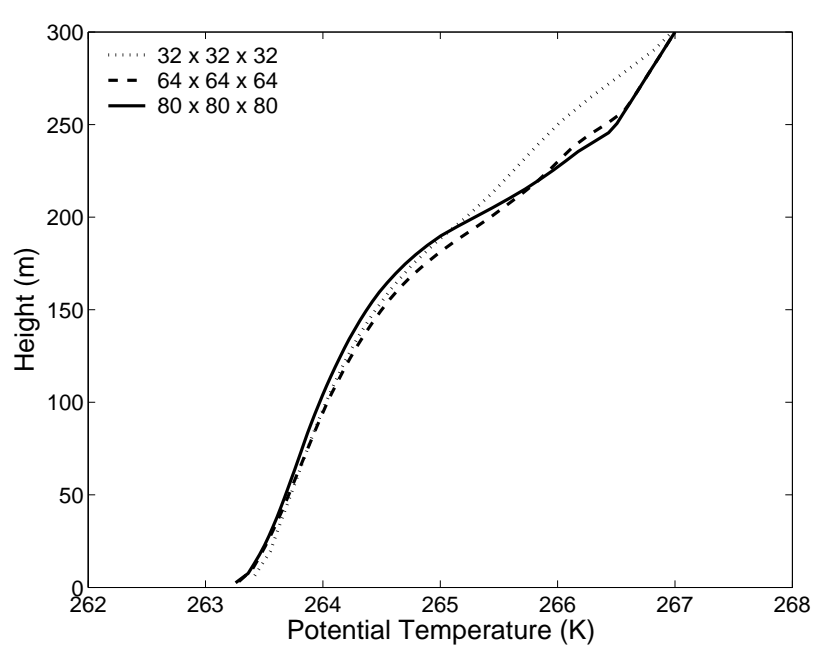

FIG. 4. Mean temperature profiles. These profiles are averaged over the last one hour of simulation.

top of the boundary layer and become more than 1 in the inversion layer.

It is straightforward to show that the ratio between $R i_{g}$ and $R i_{f}$ is the turbulent Prandtl number $\left(\operatorname{Pr}_{t}\right)$ (Derbyshire 1999; Howell and Sun 1999):

$$
P r_{t}=\frac{K_{M}}{K_{H}}=\frac{R i_{g}}{R i_{f}}
$$

where $K_{M}$ and $K_{H}$ represent eddy diffusivities for momentum and heat flux, respectively. The dependence of $P r_{t}$ on atmospheric stability is not strong (Derbyshire 1999; Howell and Sun 1999). Inside the boundary layer (up to $\sim 150$ m), (almost) all our simulated results yield $\frac{R i_{g}}{R i_{f}}=P r_{t} \sim 0.7$ (not shown here). Based on phenomenological theories of turbulence Townsend (1976) and Yakhot and Orszag (1986) also derived $P r_{t}=0.7$. However, in the surface layer our results show that the values of $P r_{t}$ increase to $\sim 1$. This is consistent with 'Microfronts' field experimental data analyzed by Howell and Sun (1999). They found on average, the estimates of $P r_{t}$ at $3 \mathrm{~m}$ level are higher than at the $10 \mathrm{~m}$ level, indicating that the relative efficiency of turbulent momentum transfer with respect to heat transfer increases in the proximity of the land surface (Howell and Sun 1999).

In SBL simulations, one can test the performance of a SGS model by plotting a local nondimensional shear:

$$
\Phi_{M L}=\frac{\kappa z}{u_{* L}} \sqrt{\left(\frac{\partial U}{\partial z}\right)^{2}+\left(\frac{\partial V}{\partial z}\right)^{2}}
$$

and nondimensional temperature gradient:

$$
\Phi_{H L}=\frac{\kappa z}{\theta_{* L}} \frac{\partial \Theta}{\partial z}
$$

as a function of local stability parameter $(z / \Lambda)$ and comparing with field-observations-based formulations. Here, $\Lambda$ 

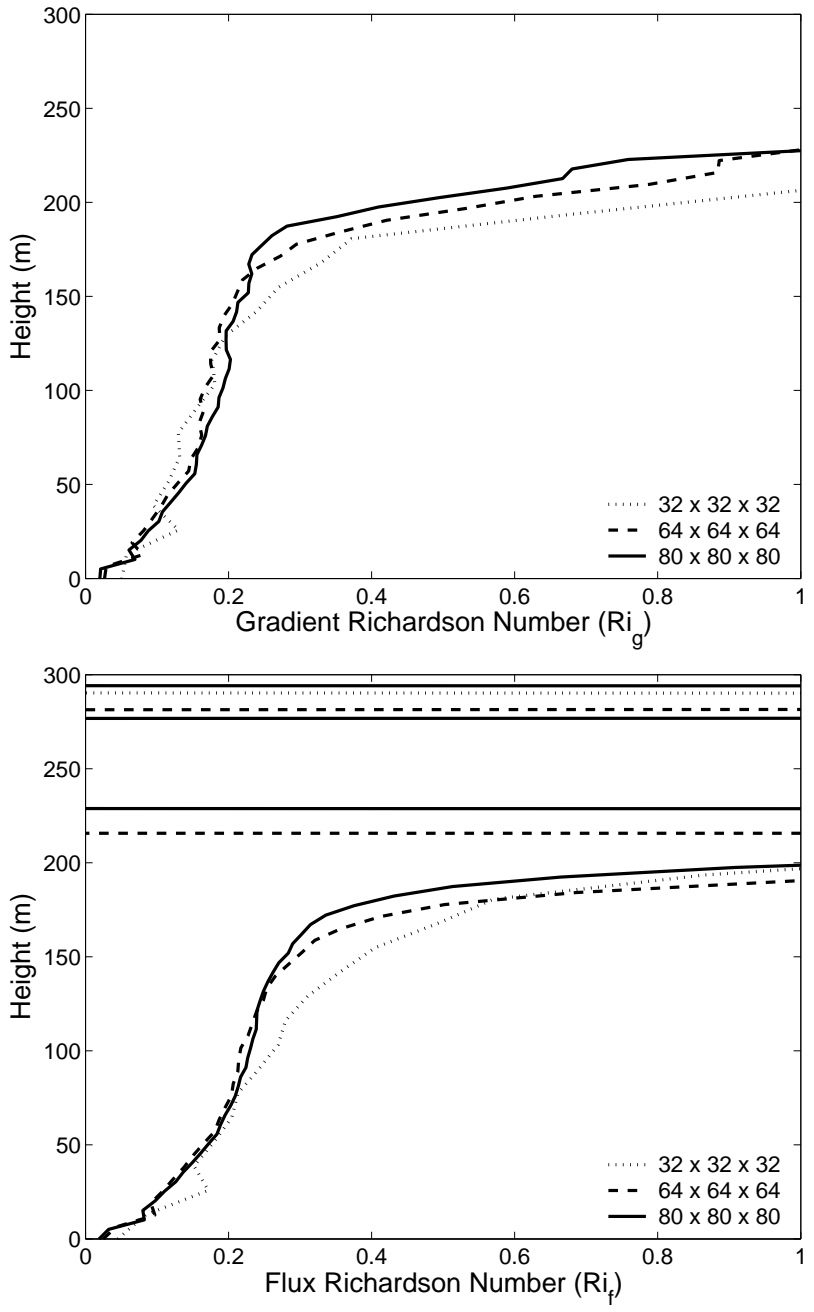

FIG. 5. Mean profiles of gradient Richardson number (top) and flux Richardson number (bottom). These profiles are averaged over the last one hour of simulation.

denotes the local Obukhov length. In this work, a subscript ' $L$ ' on the turbulence quantities (e.g., $u_{* L}$ ) will be used to specify evalutation using local turbulence quantities - otherwise, surface values are implied. Recently, Mahrt and Vickers (2003) called this type of similarity theory 'hybrid similarity theory', since it approaches Monin-Obukhov similarity as $z$ decreases and also conforms to z-less stratification as $z \rightarrow \infty$. In Figure 6 we plot the 'hybrid' nondimensionalized gradients and compare them with the formulations by Businger et al. (1971):

$$
\begin{gathered}
\Phi_{M L}=1+4.7 \frac{z}{\Lambda} \\
\Phi_{H L}=0.74+4.7 \frac{z}{\Lambda},
\end{gathered}
$$

and by Beljaars and Holtslag (1991):

$$
\Phi_{M L}=1+\frac{z}{\Lambda}\left[a+b e^{-d \frac{z}{\Lambda}}\left(1+c-d \frac{z}{\Lambda}\right)\right]
$$
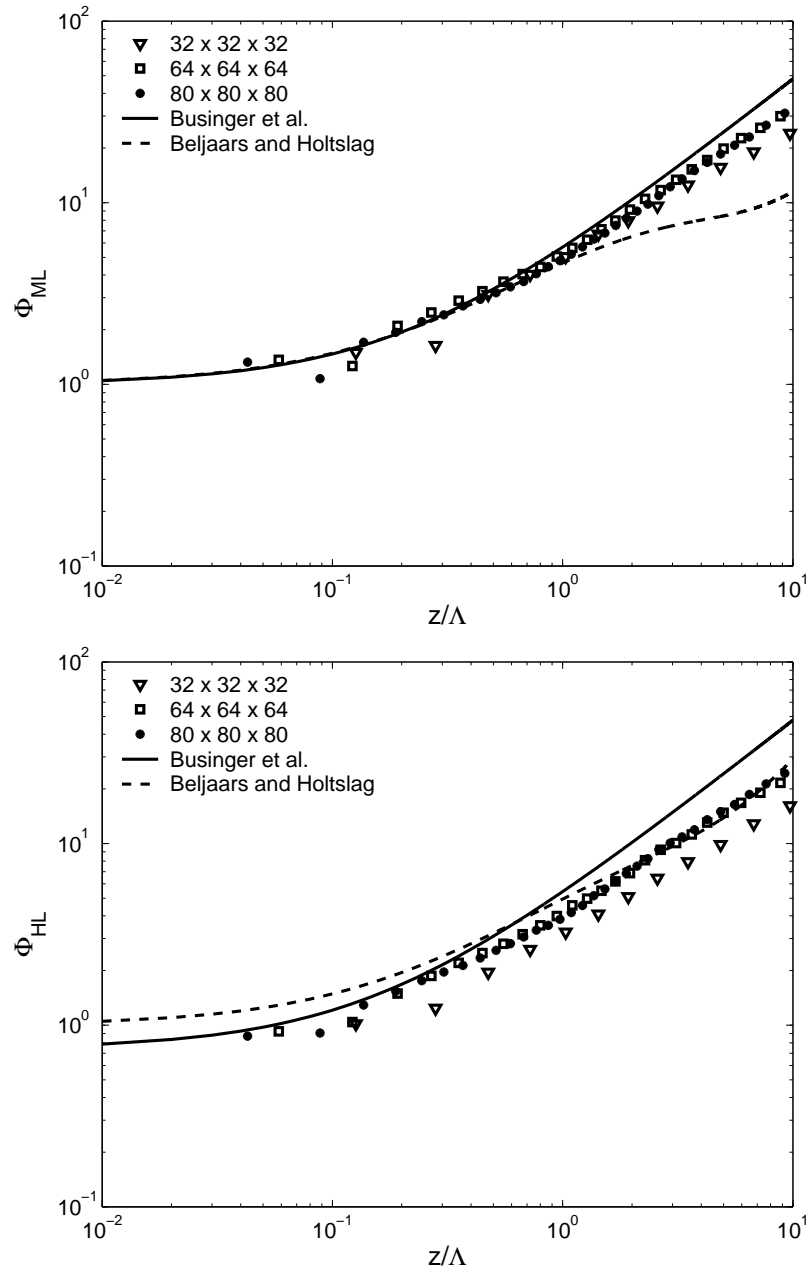

FIG. 6. Locally computed nondimensional gradients of velocity (top) and temperature (bottom) against the local stability parameter. These statistics are computed during the last one hour of simulation. The field-observations-based formulations given by Businger et al. (1971) and Beljaars and Holtslag (1991) are also shown.

$\Phi_{H L}=1+\frac{z}{\Lambda}\left[a\left(1+\frac{2}{3} \frac{a z}{\Lambda}\right)^{1 / 2}+b e^{-d \frac{z}{\Lambda}}\left(1+c-d \frac{z}{\Lambda}\right)\right]$,

where the suggested values of the coefficients are (Beljaars and Holtslag 1991): $a=1, b=2 / 3, c=5$ and $d=0.35$. Interestingly, both these simulated gradients plotted against $z / \Lambda$ show slopes slightly smaller than the widely used Businger et al.'s formulations. Based on CASES99 field observations data, Mahrt and Vickers (2003) found a slope of 3.7 [in contrast to 4.7 as proposed by Businger et al. (1971)], which also fits our LES results remarkably well. Previous studies, such as Beljaars and Holtslag (1991) also found that $\Phi_{M L}$ and $\Phi_{H L}$ increase slower than Businger et al.'s formulations and they proposed the aforementioned nonlinear formulation (Beljaars and Holtslag 1991). 

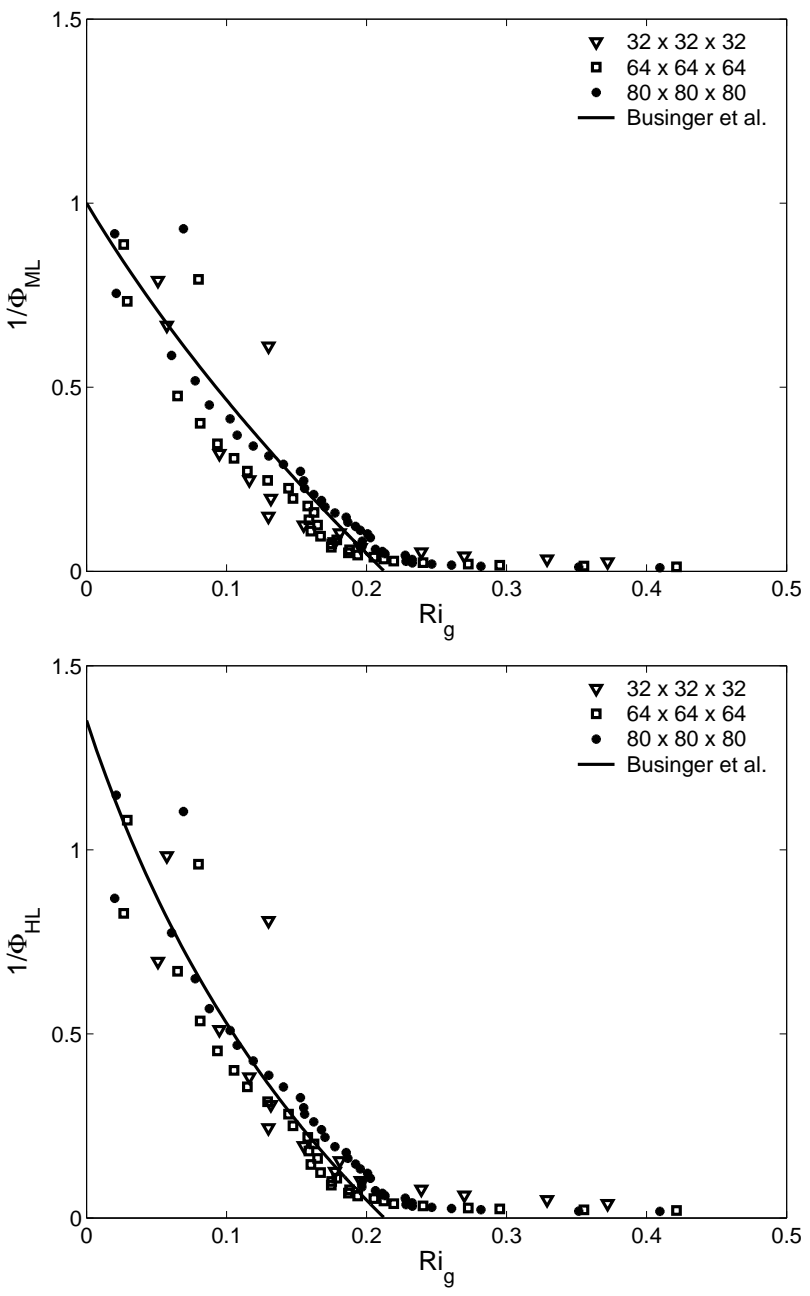

FIG. 7. Locally computed nondimensional gradients of velocity (top) and temperature (bottom) against local gradient Richardson number. These statistics are computed during the last one hour of simulation. The formulations given by Businger et al. (1971) are also shown.

There exists another representation for the nondimensional gradients in terms of local gradient Richardson number $\left(R i_{g}\right)$. Figure 7 once again shows that the agreement between our LES results and established formulations are quite satisfactory. In the literature, usually the critical gradient Richardson number $\left(R i_{g c}\right)$ is considered to be around 0.25 . For $R i_{g c}>0.25$ turbulence is very weak (Stull 1988; Brown et al. 1994), which is also noticeable in Figure 7.

\section{3) SECOND-ORder Statistics}

The mean profiles of vertical momentum flux and buoyancy flux averaged over the final hour (8-9 hours) of simulation are given in Figure 8. The dashed lines show the resolved flux, and the dotted lines denote the SGS contribution to the flux. As would be anticipated, near the ground
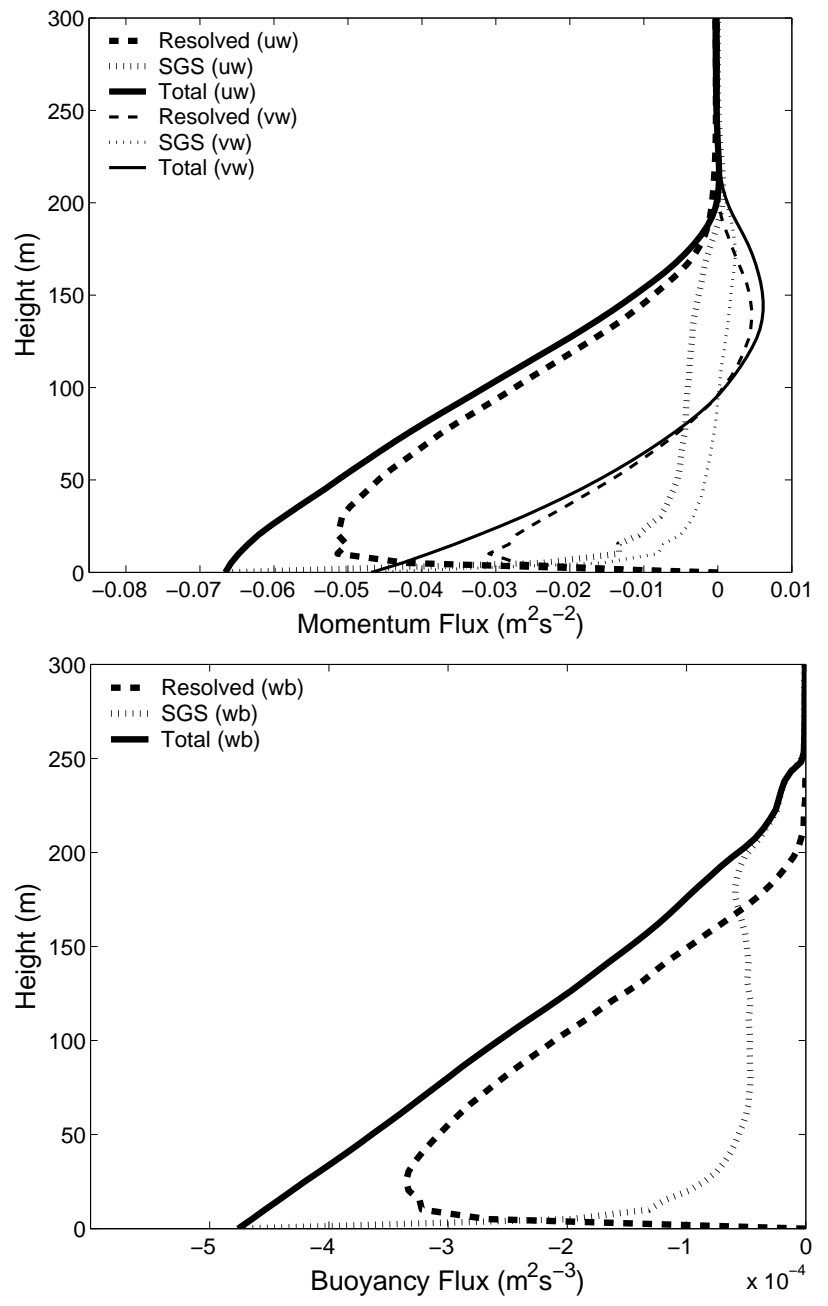

FIG. 8. Mean momentum flux (top) and buoyancy flux (bottom) profiles corresponding to the $80^{3}$ simulation using the locallyaveraged scale-dependent dynamic (LASDD) SGS model. These profiles are averaged over the last one hour of simulation.

the SGS contribution is much larger than its resolved counterpart. In the GABLS intercomparison study, there is a significant spread between the total momentum and buoyancy flux profiles simulated with different models. In particular at the surface the mean momentum and buoyancy fluxes range from 0.06 to $0.08 \mathrm{~m}^{2} \mathrm{~s}^{-2}$ and -3.5 to $-5.5 \times 10^{-4} \mathrm{~m}^{2} \mathrm{~s}^{-3}$, respectively (Beare et al. 2004). Our simulated results also fall in these ranges.

Perhaps more interesting is to explore the normalized flux profiles shown in Figure 9. Nieuwstadt's analytical model predictions are as follows (Nieuwstadt 1985):

$$
\begin{aligned}
& \frac{u_{* L}^{2}}{u_{*}^{2}}=(1-z / H)^{3 / 2} \\
& \frac{\left\langle w b_{L}\right\rangle}{\left\langle w b_{s}\right\rangle}=(1-z / H) .
\end{aligned}
$$



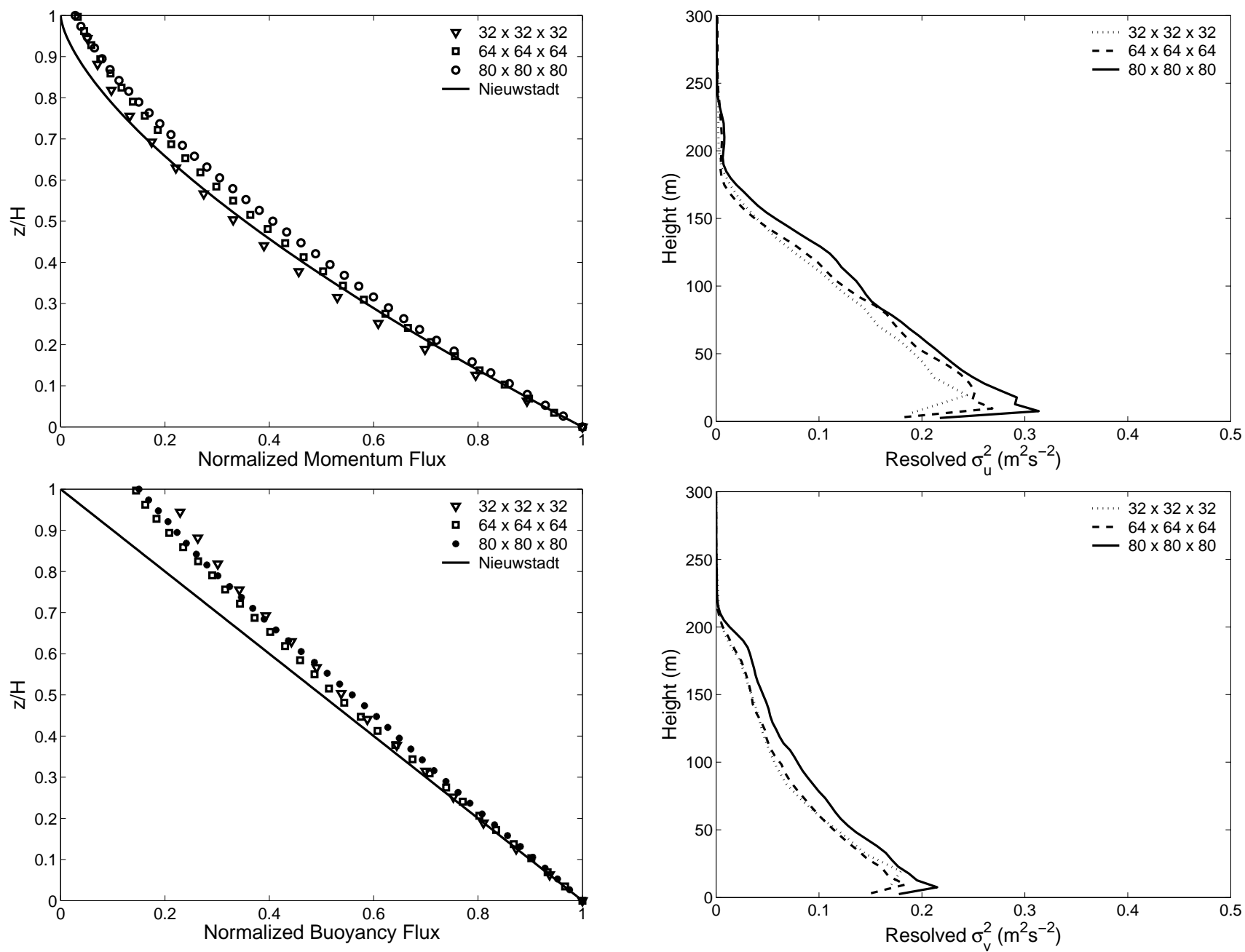

FIG. 9. Mean normalized momentum flux (top) and normalized buoyancy flux (bottom) profiles from the simulations performed in the present work. These profiles are averaged over the last one hour of simulation. Theoretical predictions by Nieuwstadt (1985) are also shown for comparison.

Our model simulated results are in close agreement with Nieuwstadt's predictions.

Figures 10 and 11 show the (resolved) variances of velocity components and temperature. In the surface layer, the normalized resolved velocity variances are smaller than Nieuwstadt's field observations (Nieuwstadt 1984a, b). This is expected as the SGS contributions to these variances have not been added here. This will be done while studying Nieuwstadt's local scaling hypothesis and will be shown to be in excellent agreement with Nieuwstadt's observations (see below). We would like to point out that in contrast to our simulated results, the nonlinear SGS model simulations by Kosović and Curry (2000) surprisingly yielded surface layer velocity variances (resolved plus SGS) which were $\sim 40$ percent smaller than Nieuwstadt's observations.

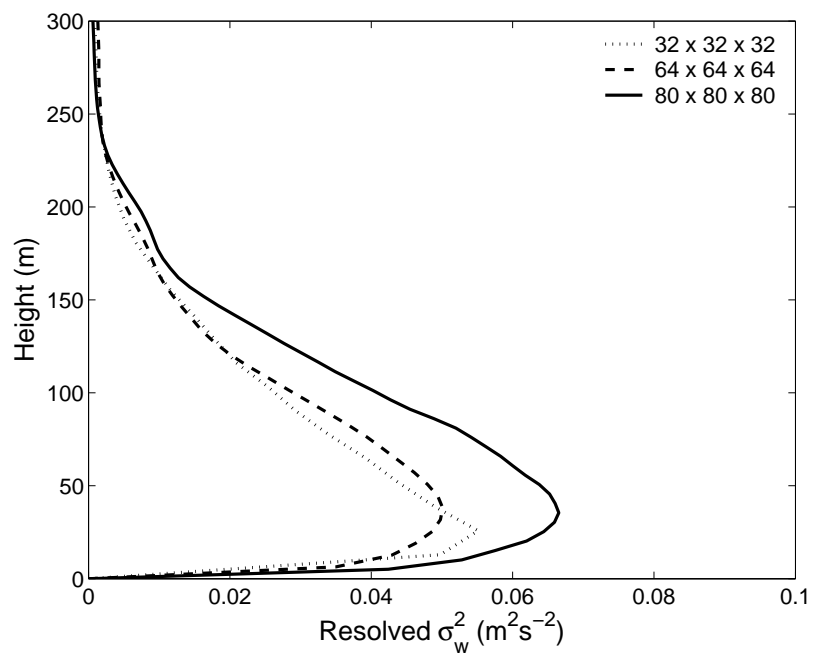

FIG. 10. Resolved velocity variances from the simulations performed in the present work. These profiles are averaged over the last one hour of simulation.

In his local scaling hypothesis, Nieuwstadt (Nieuwstadt 


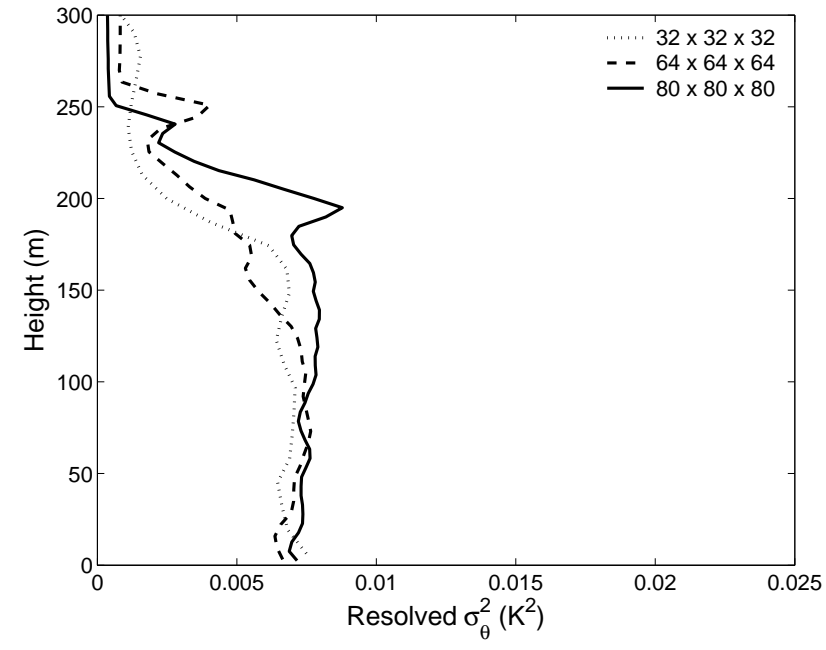

FIG. 11. Resolved temperature variances from the simulations performed in the present work. These profiles are averaged over the last one hour of simulation.

1984a, b, 1985) conjectured that under stable stratification, the local Obukhov length $(\Lambda)$ based on local turbulent fluxes should be considered as a more fundamental length scale. Then, according to this hypothesis, dimensionless combinations of turbulent variables [gradients, fluxes, (co-)variances etc.] which are measured at the same height $(z)$ could be expressed as 'universal' functions of the stability parameter, $\zeta(=z / \Lambda)$. Exact forms of these functions could be predicted by dimensional analysis only in the asymptotic very stable case $(\zeta \rightarrow \infty)$, as discussed below. In the very stable regime (z-less condition), since any explicit dependence on $z$ disappears, local scaling predicts that dimensionless turbulent quantities asymptotically approach constant values (Nieuwstadt 1984a, b, 1985). Local scaling could be viewed as a generalization of the well established Monin-Obukhov (M-O) similarity theory (Monin and Yaglom 1971; Sorbjan 1989). M-O similarity theory is strictly valid in the surface layer (lowest $10 \%$ of the ABL), whereas local scaling describes the turbulent structure of the entire SBL Nieuwstadt 1984a, b, 1985).

Whether or not our LES-generated statistics support the local scaling hypothesis was studied extensively in our recent work (Basu et al. 2005). In that study, we also performed rigorous statistical analyses of field observations and wind-tunnel measurements in order to verify the validity of local scaling hypothesis under very stable conditions. An extensive set of turbulence statistics, computed from field and wind-tunnel measurements and also from LES-generated datasets, supported the validity of the local scaling hypothesis (in the cases of traditional bottom-up as well as upsidedown stable boundary layers over homogeneous, flat terrains). We demonstrated that non-turbulent effects need to be removed from field data while studying similarity hy-
TABLE 2. Number of samples in each stability class.

\begin{tabular}{lcccc}
\hline \hline Class & Stability $(\zeta)$ & $32^{3}$ & $64^{3}$ & $80^{3}$ \\
\hline S1 & $0.00-0.10$ & 0 & 1 & 2 \\
S2 & $0.10-0.25$ & 1 & 2 & 3 \\
S3 & $0.25-0.50$ & 2 & 3 & 3 \\
S4 & $0.50-1.00$ & 1 & 4 & 6 \\
S5 & $>1.00$ & 8 & 11 & 14 \\
\hline
\end{tabular}

potheses, otherwise the results could be misleading (Basu et al. 2005). For completeness of the present paper, we decided to include some key local scaling results. For ease in representation, we categorize our LES-generated database based on local stabilities $(z / \Lambda)$ (see Table 2$)$. The class S1 represents near neutral stability; while S5 corresponds to the very stable regime.

In Figures 12 and 13 we plot the normalized standard deviation of velocity components and temperature, respectively. The results are presented using standard boxplot notation with marks at $95,75,50,25$, and 5 percentile of an empirical distribution. The SGS contributions to the total standard deviations are estimated following the approach of Mason (Mason 1989; Mason and Derbyshire 1990).

It is quite evident from Figures 12 and 13 that the normalized standard deviation of the turbulence variables closely follows the local scaling predictions and also z-less stratification. In Table 3 we further report the median values of the turbulence statistics corresponding to the category S5. Loosely, these median values could be considered as the asymptotic z-less values, which are found to be remarkably close to Nieuwstadt's analytical predictions and also field observations (see Table 3). For an example, Nieuwstadt's theory predicts that the normalized vertical velocity standard deviation asymptotically approaches $\sim 1.4$ in the $\mathrm{z}-$ less regime. In Basu et al. (2005), we observed this value to be in the narrow range of 1.4 to 1.6. Recently, Heinemann (2004) compiled a list (see Table 2 of their paper) of turbulence statistics under very stable conditions $\left(\zeta_{\max } \sim 25\right)$ reported by various researchers. They found an asymptotic value of $\sim 1.6$ for $\sigma_{w} / u_{* L}$, in accord with Sorbjan (1986).

In Figure 14, we report the mutual correlations between $u, w$ and $\theta$. The z-less values are also reported in Table 3. Once again, these values are very similar to the ones compiled by Heinemann (2004), our previous study (Basu et al. 2005), results of Sorbjan (1986) and theoretical predictions of Nieuwstadt (1984b). As a note, Kaimal and Finnigan (1994) also report that for $0<\zeta<1, r_{u \theta}=0.6$, which is close to the values found in the present study.

In light of the foregoing analyses it is certain that the local scaling hypothesis of Nieuwstadt, which has survived the last two decades, still holds for a wide range of stabilities and is well reproduced by our LES model. 

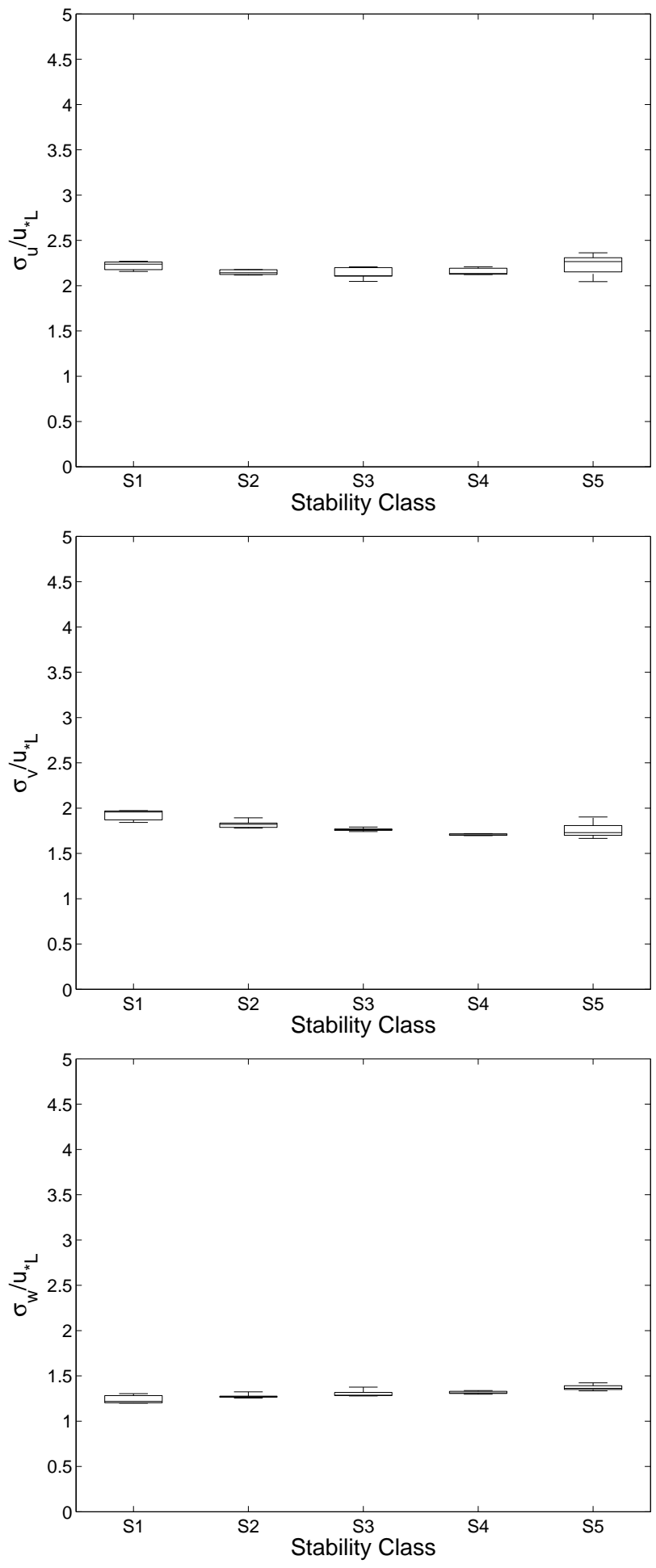

FIG. 12. Normalized longitudinal (top), transverse (middle), and vertical (bottom) velocity standard deviations. These statistics are averaged over the last one hour of simulation.

\section{4) SGS COEFFICIENTS}

Figures 15 and 16 show the SGS coefficients: $C_{S}, P r_{S G S}$, and the averaged scale-dependent parameters: $\beta, \beta_{\theta}$, dynam-

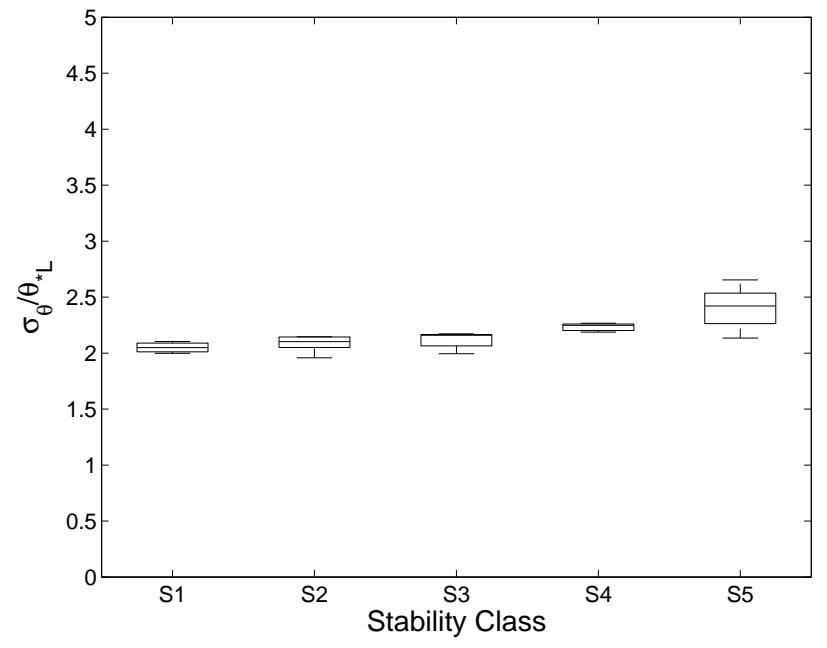

FIG. 13. Normalized temperature standard deviations. This statistic is averaged over the last one hour of simulation.

ically obtained using the locally-averaged scale-dependent dynamic model. $\beta$ and $\beta_{\theta}$ are found to be significantly smaller than 1 in the entire boundary layer. This stresses the fact that the assumption of scale-invariance in anisotropic stably stratified flows is not appropriate. Indeed, both $C_{S}$ and $\operatorname{Pr}_{S G S}$ are found to be scale dependent. The scaledependent parameters are also expected to depend on local stability as they decrease significantly in the strongly stratified inversion layer. $C_{S}$ is found to decrease with increasing atmospheric stability, consistent with recent field observations (Porté-Agel et al. 2001; Kleissl et al. 2003). The SGS Prandtl number $\left(P r_{S G S}\right)$ is more or less constant inside the boundary layer and gradually increases to $\sim 1$ in the inversion layer, as commonly assumed. Moreover, the values of $P r_{S G S}$ increase in the surface layer. Earlier, we described very similar behavior in the case of turbulent Prandtl number $\left(\operatorname{Pr}_{t}\right)$.

TABLE 3. z-less values of turbulence statistics.

\begin{tabular}{lccc}
\hline \hline $\begin{array}{l}\text { Turbulence } \\
\text { Statistics }\end{array}$ & $\begin{array}{c}\text { Large-eddy } \\
\text { Simulations }\end{array}$ & $\begin{array}{c}\text { Nieuwstadt } \\
(1984 \mathrm{~b}, 1985)\end{array}$ & $\begin{array}{c}\text { Sorbjan } \\
(1986)\end{array}$ \\
\hline$\sigma_{u} / u_{* L}$ & 2.3 & 2.0 & 2.4 \\
$\sigma_{v} / u_{* L}$ & 1.7 & 1.7 & 1.8 \\
$\sigma_{w} / u_{* L}$ & 1.4 & 1.4 & 1.6 \\
$\sigma_{\theta} / \theta_{* L}$ & 2.4 & 3.0 & 2.4 \\
$r_{u w}$ & -0.32 & - & - \\
$r_{u \theta}$ & 0.56 & - & 0.50 \\
$r_{w \theta}$ & -0.30 & -0.24 & - \\
\hline
\end{tabular}



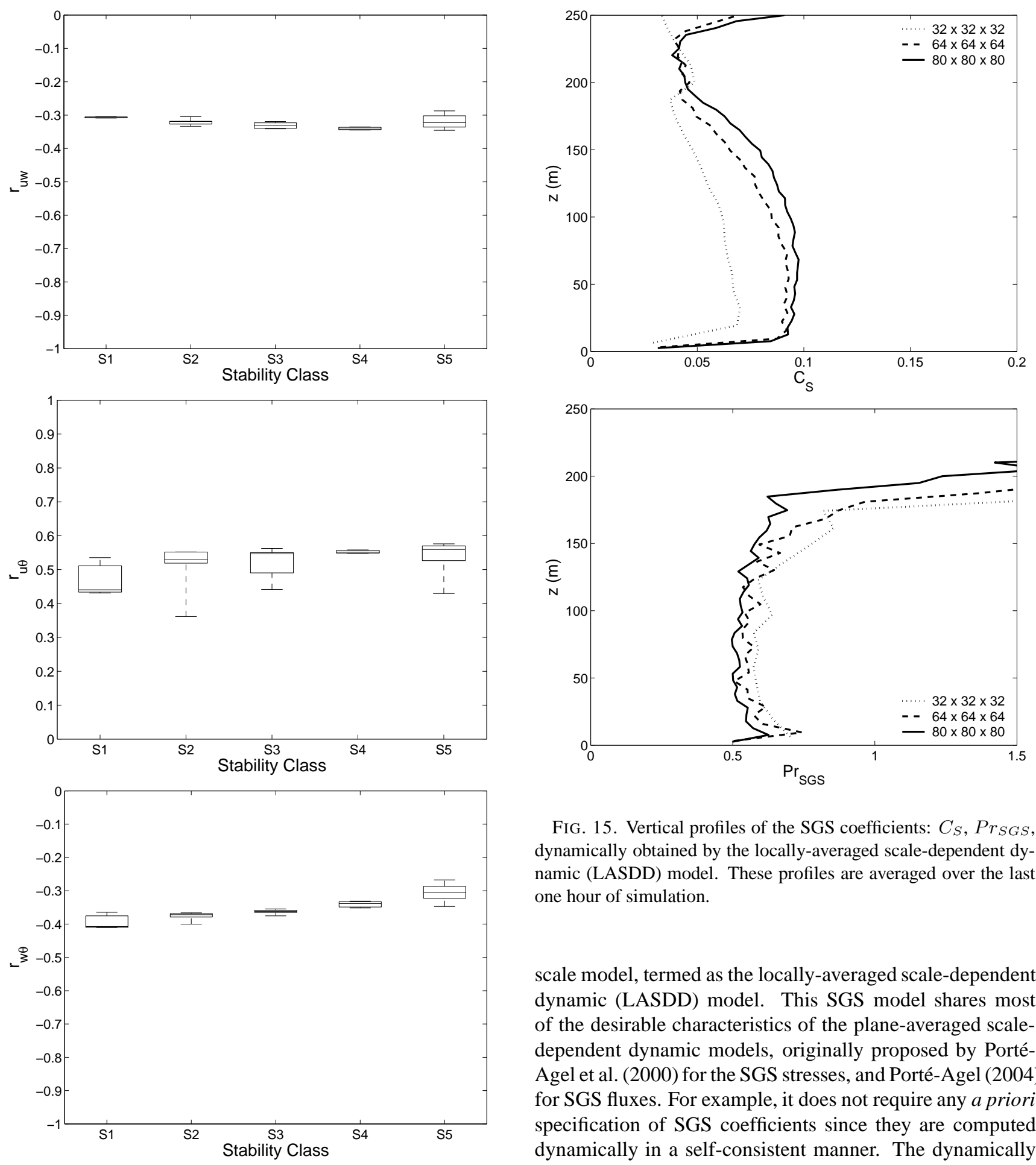

FIG. 14. Correlation between $u$ and $w\left(r_{u w}\right)$ (top), $u$ and $\theta\left(r_{u \theta}\right)$ (middle), and $w$ and $\theta\left(r_{w \theta}\right)$ (bottom). These statistics are averaged over the last one hour of simulation.

\section{Concluding Remarks and Future Perspectives}

One of the contributions of this research is the development and implementation of a new-generation subgrid-

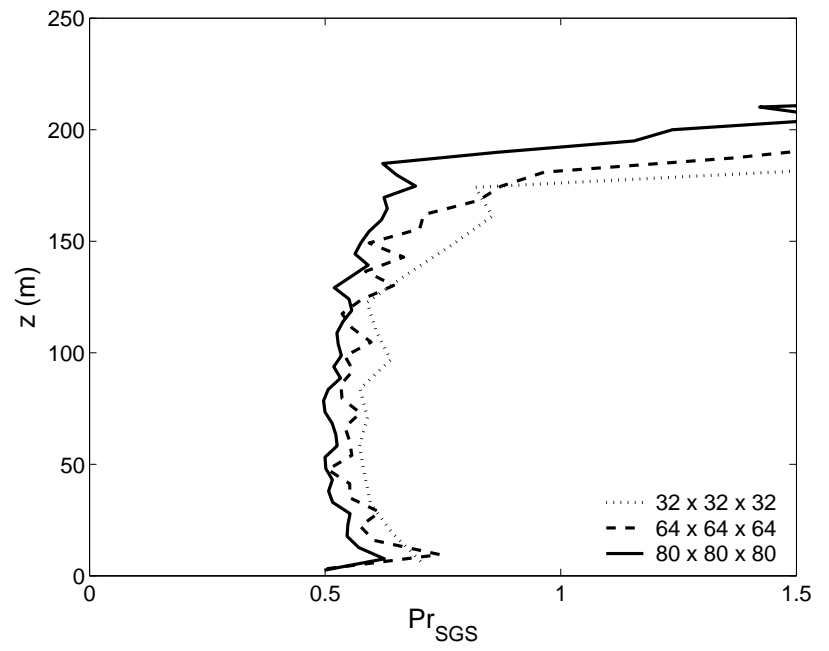

FIG. 15. Vertical profiles of the SGS coefficients: $C_{S}, P r_{S G S}$, dynamically obtained by the locally-averaged scale-dependent dynamic (LASDD) model. These profiles are averaged over the last one hour of simulation.

scale model, termed as the locally-averaged scale-dependent dynamic (LASDD) model. This SGS model shares most of the desirable characteristics of the plane-averaged scaledependent dynamic models, originally proposed by PortéAgel et al. (2000) for the SGS stresses, and Porté-Agel (2004) for SGS fluxes. For example, it does not require any a priori specification of SGS coefficients since they are computed dynamically in a self-consistent manner. The dynamically estimated coefficients are found to strongly depend on filter scale and atmospheric stability, in close agreement with a priori field studies (Porté-Agel et al. 2001; Kleissl et al. 2003). However, in contrast to the original plane-averaged version, the LASDD model does not suffer from the insufficient SGS dissipation problem in simulations of stable boundary layers.

The potential of our SGS model is made clear in coarse- 

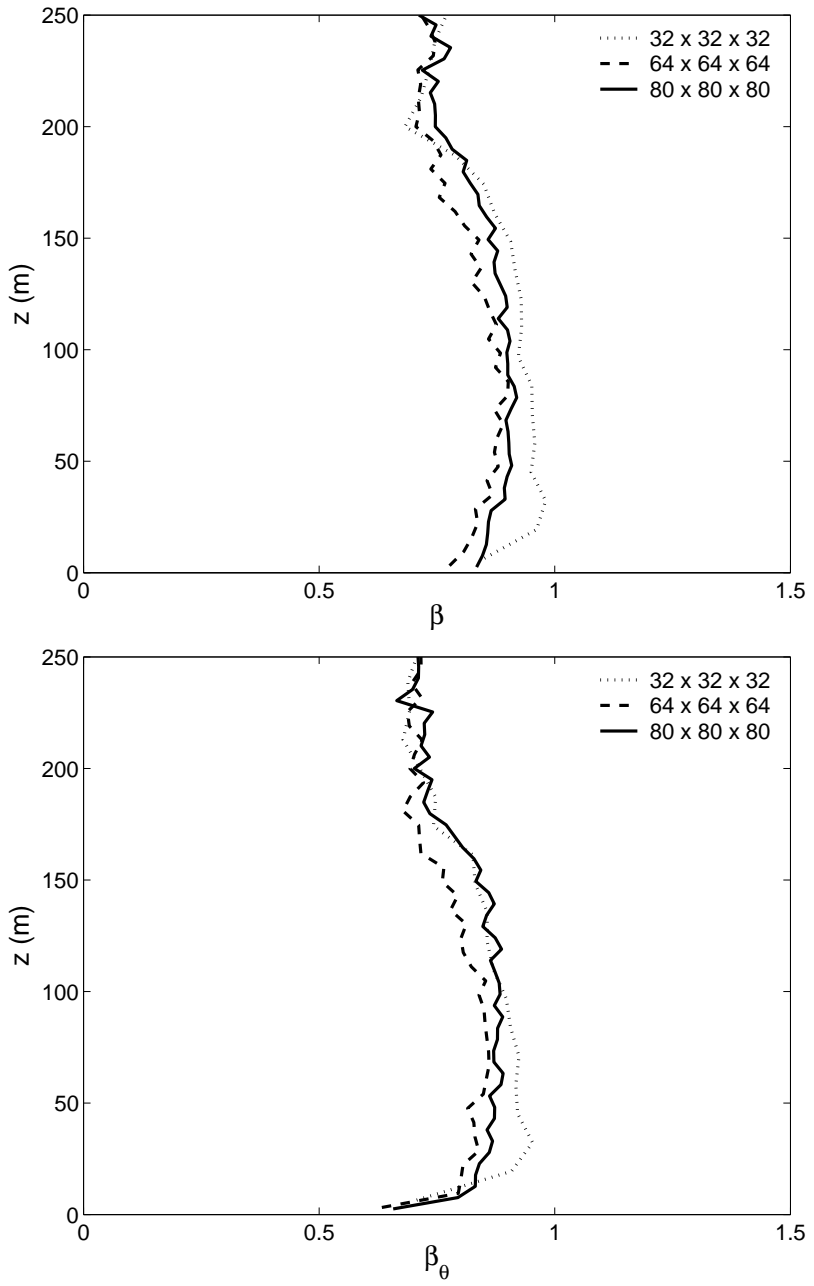

FIG. 16. Vertical profiles of the scale-dependent parameters: $\beta$, $\beta_{\theta}$, dynamically obtained by the locally-averaged scale-dependent dynamic (LASDD) model. These profiles are averaged over the last one hour of simulation.

resolution large-eddy simulations of moderately stable boundary layers. Overall, the agreements between our LESgenerated turbulence statistics and observations, as well as some well-established empirical formulations and theoretical predictions are remarkable. The results also show clear improvements over most of the traditional SGS models in the surface layer. In essence, we showed that tuning-free simulations of stable atmospheric boundary layers are feasible even with relatively coarse resolutions if one uses a robust and reliable SGS scheme.

The next logical step would be to check the performance of this new-generation SGS scheme in simulating very stable boundary layers. This would of course require extensive validation against existing profiles of various turbulence statistics measured during different field campaigns (e.g, Cooperative Atmosphere-Surface Exchange Study 1999 -
CASES99; Beaufort Sea Arctic Stratus Experiment - BASE; Surface Heat Budget of the Arctic Ocean - SHEBA). One of the characteristics of strongly stratified boundary layers is the existence of global intermittency (turbulent burstings in the midst of a laminar flow). In contrast to the traditional SGS models, the locally-averaged scale-dependent dynamic LES model has the correct behavior in laminar and transitional flows. This makes us believe that this model will be able to model the complex intermittency behavior of the very stable boundary layer flows.

Contemporary SBL research has revealed that in very stable regimes the relative importance of radiative cooling and heat exchange with the underlying soil becomes as significant as turbulent mixing (van de Wiel 2002). This means that radiation and soil physics should also be included in LES models before attempting very stable simulations. Another interesting feature of this type of boundary layer flows is the presence of gravity waves. Conceptually, LES is capable of simulating gravity waves, provided the domain size is large enough. Unfortunately, the present computational power dictates that in such cases one must have a relatively coarse resolution making thus the locally-averaged scale-dependent dynamic model more desirable than the laminarization-prone traditional SGS models. These issues will be addressed in our future research.

Acknowledgments. The authors are grateful to Rob Stoll for his generous help during the course of this work. We thank Efi Foufoula-Georgiou for many thought provoking discussions. This work was partially funded by NSF and NASA grants. All the computational resources were kindly provided by the National Center for Atmospheric Research (NCAR).

\section{REFERENCES}

Albertson, J. D., and M. B. Parlange, 1999: Natural integration of scalar fluxes from complex terrain. Adv. Wat. Res., 23, 239-252.

Andrén, A., 1995: The structure of stably stratified atmospheric boundary layers: A large-eddy simulation study. Quart. J. Roy. Meteorol. Soc., 121, 961-985.

Andrén, A., A. R. Brown, J. Graf, P. J. Mason, C.-H. Moeng, F. T. M. Nieuwstadt, and U. Schumann, 1994: Large-eddy simulation of a neutrally stratified boundary layer: A comparison of four computer codes. Quart. J. Roy. Meteorol. Soc., 120, $1457-1484$.

Arya, S. P., 2001: Introduction to Micrometeorology. Academic Press, 420 pp.

Basu, S., F. Porté-Agel, E. Foufoula-Georgiou, J.-F. Vinuesa, and M. Pahlow, 2005: Revisiting the local scaling hypothesis in stably stratified atmospheric boundary layer turbulence: an integration of field and laboratory measurements with large-eddy simulations. Boundary-Layer Meteorol. Under review.

Beare, R. J., and M. K. MacVean, 2004: Resolution sensitivity and scaling of large-eddy simulations of the stable boundary layer. Boundary-Layer Meteorol., 112, 257-281. 
Beare, R. J., et al., 2004: An intercomparison of large-eddy simulations of the stable boundary layer. Boundary-Layer Meteorol. Under review.

Beljaars, A. C. M., and A. A. M. Holtslag, 1991: Flux parameterization over land surfaces for atmospheric models. J. Appl. Meteorol., 30, 327-341.

Brost, R. A., and J. C. Wyngaard, 1978: A model study of the stably stratified planetary boundary layer. J. Atmos. Sci., 35, 1427-1440.

Brown, A. R., S. H. Derbyshire, and P. J. Mason, 1994: Large-eddy simulation of stable atmospheric boundary layers with a revised stochastic subgrid model. Quart. J. Roy. Meteorol. Soc., 120, 1485-1512.

Businger, J. A., J. C. Wyngaard, Y. Izumi, and E. F. Bradley, 1971: Flux-profile relationships in the atmospheric surface layer. $J$. Atmos. Sci., 28, 181-189.

Canuto, C., M. Y. Hussaini, A. Quarteroni, and T. A. Zhang, 1988: Spectral Methods in Fluid Dynamics. Springer Verlag, 557 pp.

Canuto, V. M., and Y. Cheng, 1997: Determination of the Smagorinsky-Lilly constant $C_{S}$. Phys. Fluids, 9, 1368-1378.

Deardorff, J. W., 1970: Convective velocity and temperature scales for the unstable planetary boundary layer and Rayleigh convection. J. Atmos. Sci., 27, 1211-1213.

Deardorff, J. W., 1972: Theoretical expression for the countergradient vertical heat flux. J. Geophys. Res., 77, 5900-5904.

Deardorff, J. W., 1974: Three-dimensional numerical study of turbulence in an entraining mixed layer. Boundary-Layer Meteorol., 7, 199-226.

Deardorff, J. W., 1980: Stratocumulus-capped mixed layers derived from a three-dimensional model. Boundary-Layer Meteorol., 18, 495-527.

Derbyshire, S. H., 1990: Nieuwstadt's stable boundary layer revisited. Quart. J. Roy. Meteorol. Soc., 116, 127-158.

Derbyshire, S. H., 1999: Stable boundary-layer modelling: Established approaches and beyond. Boundary-Layer Meteorol., 90, 423-446.

Germano, M., 1992: Turbulence: the filtering approach. J. Fluid Mech., 238, 325-336.

Germano, M., U. Piomelli, P. Moin, and W. H. Cabot, 1991: A dynamic subgrid-scale eddy viscosity model. Phys. Fluids A, 3, 1760-1765.

Geurts, B. J., 2003: Elements of Direct and Large-eddy Simulation. Edwards, 329 pp.

Ghosal, S., T. S. Lund, P. Moin, and K. Akselvoll, 1995: A dynamic localization model for large-eddy simulation of turbulent flows. Phys. Fluids A, 3, 1760-1765.

Heinemann, G., 2004: Local similarity properties of the continuously turbulent stable boundary layer over Greenland. Boundary-Layer Meteorol., 112, 283-305.

Higgins, C., M. B. Parlange, and C. Meneveau, 2003: Alignment trends of velocity gradients and subgrid scale fluxes in the turbulent atmospheric boundary layer. Boundary-Layer Meteorol., 109, 59-83.

Holtslag, A. A. M., 2003: GABLS initiates intercomparison for stable boundary layer case. GEWEX News, 13, 7-8.

Howell, J. F., and J. Sun, 1999: Surface-layer fluxes in stable conditions. Boundary-Layer Meteorol., 90, 495-520.
Hunt, J. C. R., D. D. Stretch, and R. E. Britter, 1988: Length scales in stably stratified turbulent flows and their use in turbulence models. Stably Stratified Flows and Dense Gas Dispersion, J. S. Puttock, Ed., Clarendon Press, pp. 285-321.

Hunt, J. C. R., G. J. Shutts, and S. H. Derbyshire, 1996: Stably stratified flows in meteorology. Dyn. Atmos. and Oceans, 23, 63-79.

Kaimal, J. C., and J. J. Finnigan, 1994: Atmospheric Boundary Layer Flows: Their Structure and Measurement. Oxford University Press, $289 \mathrm{pp}$.

Kleissl, J., C. Meneveau, and M. B. Parlange, 2003: On the magnitude and variability of subgrid-scale eddy-diffusion coefficients in the atmospheric surface layer. J. Atmos. Sci., 60, 2372-2388.

Kleissl, J., M. B. Parlange, and C. Meneveau, 2004: Field experimental study of dynamic Smagorinsky models in the atmospheric surface layer. J. Atmos. Sci., 61, 2296-2307.

Kosović, B., 1997: Subgrid-scale modelling for the large-eddy simulation of high-Reynolds-number boundary layers. J. Fluid Mech., 338, 151-182.

Kosović, B., and J. A. Curry, 2000: A large eddy simulation study of a quasi-steady, stably stratified atmospheric boundary layer. J. Atmos. Sci., 57, 1052-1068.

Lilly, D. K., 1967: The representation of small-scale turbulence in numerical simulation experiments. Proc. IBM Scientific Computing Symposium on Environmental Sciences, pp. 195-210.

Lilly, D. K., 1992: A proposed modification of the Germano subgrid-scale closure method. Phys. Fluids A, 4, 633-635.

Mahrt, L., 1998: Stratified atmospheric boundary layers and breakdown of models. Theoret. Comput. Fluid Dyn., 11, 263-279.

Mahrt, L., and D. Vickers, 2003: Formulation of turbulent fluxes in the stable boundary layer. J. Atmos. Sci., 60, 2538-2548.

Mason, P., 1989: Large-eddy simulation of the convective atmospheric boundary layer. J. Atmos. Sci., 46, 1492-1516.

Mason, P., 1994: Large-eddy simulation: A critical review of the technique. Quart. J. Roy. Meteorol. Soc., 120, 1-26.

Mason, P. J., and A. R. Brown, 1999: On subgrid models and filter operations in large-eddy simulations. J. Atmos. Sci., 56, 21012114.

Mason, P. J., and S. H. Derbyshire, 1990: Large-eddy simulation of the stably-stratified atmospheric boundary layer. BoundaryLayer Meteorol., 53, 117-162.

Meneveau, C., and J. Katz, 2000: Scale-invariance and turbulence models for large-eddy simulation. Ann. Rev. Fluid Mech., 32, $1-32$.

Meneveau, C., T. S. Lund, and W. H. Cabot, 1996: A Lagrangian dynamic subgrid-scale model of turbulence. J. Fluid Mech., 319, 353-385.

Métais, O., and M. Lesieur, 1992: Spectral large-eddy simulations of isotropic and stably-stratified turbulence. J. Fluid Mech., 239, 157-194.

Moeng, C.-H., 1984: A large-eddy simulation model for the study of planetary boundary-layer turbulence. J. Atmos. Sci., 41, 2052-2062.

Moin, P., K. Squires, W. Cabot, and S. Lee, 1991: A dynamic subgrid-scale model for compressible turbulence and scalar transport. Phys. Fluids A, 3, 2746-2757. 
Monin, A. S., and A. M. Yaglom, 1971: Statistical Fluid Mechanics: Mechanics of Turbulence. vol. 1, MIT Press, 769 pp.

Nieuwstadt, F. T. M., 1984a: Some aspects of the turbulent stable boundary layer. Boundary-Layer Meteorol., 30, 31-55.

Nieuwstadt, F. T. M., 1984b: The turbulent structure of the stable, nocturnal boundary layer. J. Atmos. Sci., 41, 2202-2216.

Nieuwstadt, F. T. M., 1985: A model for the stationary, stable boundary layer. Turbulence and Diffusion in Stable Environments, J. C. R. Hunt, Ed., Clarendon Press, pp. 149-179.

Nieuwstadt, F. T. M., P. J. Mason, C.-H. Moeng, and U. Schumann, 1991: Large-eddy simulation of the convective boundary layer: a comparison of four computer codes. Turbulent Shear Flows 8 , F. Durst, R. Friedrich, B. E. Launder, F. W. Schmidt, U. Schumann, and J. H. Whitelaw, Eds., Springer, pp. 343-367.

Orszag, S. A., and Y.-H. Pao, 1974: Numerical computation of turbulent shear flows. Adv. Geophys., 18A, 224-236.

Piomelli, U., and J. Liu, 1995: Large-eddy simulation of rotating channel flows using a localized dynamic model. Phys. Fluids, 7, 839-848.

Porté-Agel, F., 2004: A scale-dependent dynamic model for scalar transport in LES of the atmospheric boundary layer. BoundaryLayer Meteorol., 112, 81-105.

Porté-Agel, F., C. Meneveau, and M. B. Parlange, 2000: A scaledependent dynamic model for large-eddy simulations: Application to a neutral atmospheric boundary layer. J. Fluid Mech., 415, 261-284.

Porté-Agel, F., M. B. Parlange, C. Meneveau, and W. E. Eichinger, 2001: A priori field study of the subgrid-scale heat fluxes and dissipation in the atmospheric surface layer. J. Atmos. Sci., 58, 2673-2698.

Press, W. H., B. P. Flannery, S. A. Teukolsky, and W. T. Vetterling, 1992: Numerical Recipes in Fortran. Cambridge University Press, 992 pp.

Sagaut, P., 2001: Large Eddy Simulations for Incompressible Flows. Springer-Verlag, 426 pp.

Saiki, E. M., C.-H. Moeng, and P. P. Sullivan, 2000: Largeeddy simulation of the stably stratified planetary boundary layer. Boundary-Layer Meteorol., 95, 1-30.

Sarghini, F., U. Piomelli, and B. E., 1999: Scale-similar models for large-eddy simulations. Phys. Fluids, 11, 1596-1607.

Schumann, U., 1991: Subgrid length-scales for large-eddy simulation of stratified turbulence. Theort. Comput. Fluid Dyn., 2, 279-290.

Smagorinsky, J., 1963: General circulation experiments with the primitive equations. Mon. Wea. Rev., 91, 99-164.

Sorbjan, Z., 1986: Local similarity of spectral and cospectral characteristics in the stable-continuous boundary layer. BoundaryLayer Meteorol., 35, 257-275.

Sorbjan, Z., 1989: Structure of Atmospheric Boundary Layer. Prentice-Hall, 317 pp.

Stull, R. B., 1988: An Introduction to Boundary Layer Meteorology. Kluwer Academic Publishers, 670 pp.

Sullivan, P. P., J. C. McWilliams, and C.-H. Moeng, 1994: A subgrid-scale model for large-eddy simulation of planetary boundary-layer flows. Boundary-Layer Meteorol., 71, 247-276.
Sullivan, P. P., T. W. Horst, D. H. Lenschow, C.-H. Moeng, and J. C. Weil, 2003: Structure of subfilter-scale fluxes in the atmospheric surface layer with application to large-eddy simulation modelling. J. Fluid Mech., 482, 101-139.

Townsend, A. A., 1976: The structure of turbulent shear flow. Cambridge University Press, 429 pp.

van de Wiel, B., 2002: Intermittent turbulence and oscillations in the stable boundary layer over land, Ph.D. thesis, Wageningen University, Netherlands.

Vreman, B., B. Guerts, and H. Kuerten, 1994: On the formulation of the dynamic mixed subgrid-scale model. Phys. Fluids, 6, 4057-4059.

Wong, V. C., and D. K. Lilly, 1994: A comparison of two dynamic subgrid closure methods for turbulent thermal convection. Phys. Fluids, 6, 1016-1023.

Yakhot, V., and S. A. Orszag, 1986: Renormalization group analysis of turbulence I: Basic theory. J. Sci. Comput., 1, 3-51.

Zang, Y., R. L. Street, and J. R. Koseff, 1993: A dynamic mixed subgrid-scale model and its application to turbulent recirculating flows. Phys. Fluids. A, 5, 3186-3196.

Printed November 20, 2018. 